\title{
Title: Cavitation Observations, Underwater Radiated Noise Measurements and Full-Scale Predictions of the Hydro-Spinna Turbine
}

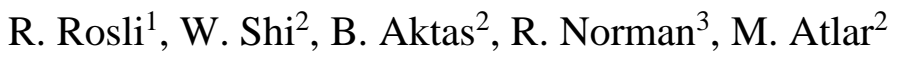 \\ ${ }^{1}$ Faculty of Engineering, Universiti Teknologi Brunei, Bandar Seri Begawan
}

BE 1410, Brunei Darussalam

${ }^{2}$ Naval Architecture, Ocean \& Marine Engineering, University of Strathclyde, Glasgow

G4 0LZ, United Kingdom

${ }^{3}$ School of Science, Agriculture \& Engineering, Newcastle University, Newcastle upon Tyne NE1 7RU, United Kingdom

*Corresponding Author: Roslynna Rosli roslynna.rosli@utb.edu.bn

Address: Faculty of Engineering, Universiti Teknologi Brunei, Tungku Highway,

BE1410, Brunei Darussalam 


\begin{abstract}
The development of marine current turbines has progressed rapidly with prototypes and full scale devices being deployed in sea. With research focusing on the hydrodynamic and design aspects of the technologies used, little is known of the impact of marine current turbine operation on marine life and environment. This paper looks at the underwater radiated noise (URN) produced from the operation of a novel tidal turbine, the Hydro-Spinna. URN measurements were taken from a $280 \mathrm{~mm}$ diameter model tested in Newcastle University. The model results were extrapolated to predict the full scale URN level for three turbine diameters of $5 \mathrm{~m}, 10 \mathrm{~m}$ and $15 \mathrm{~m}$ and compared to the fish reaction level acoustic level provided by the International Council for the Exploration of the Sea (ICES) as a reference. Analysis showed an increase in noise level with turbine diameters and that for all diameters, the highest noise levels were observed at Tip Speed Ratio $=1$ where the thrust on the turbine is at its maximum. The noise levels predicted for the Hydro-Spinna at this off-design condition is above the ICES threshold, it was found that at optimal operating conditions the noise level would be below the threshold.
\end{abstract}

Keywords: tidal turbine, tidal turbine operation, underwater radiated noise, turbine radiated noise, noise predictions 


\section{Introduction}

Tidal current turbine energy devices have been developed to extract the energy content of a tidal current. Significant developments have been achieved over recent years with the establishment of the European Marine Energy Centre (EMEC) in 2003 propelling the progress in marine energy technology further. The EMEC site in the north of Scotland is blessed with ample marine energy potential having an average tidal velocity of $3.7 \mathrm{~m} / \mathrm{s}$ (EMEC, 2013). However, tidal energy potential is location specific and not all locations in the world are gifted with such tidal energy potential. Various prototype devices have been developed with a range of energy extraction methods, however most new designs tend to employ the horizontal axis turbine concept from the already mature wind energy industry. As reported in (Rosli et al, 2016), a novel marine current turbine, the Hydro-Spinna, has been developed at Newcastle University to operate at low tidal stream velocity and therefore enable energy extraction from sites with lower resource potential.

Marine current turbines extract the kinetic energy content of the tides and convert it to useful electrical energy. Marine current turbines, like other renewable energy converting devices are more environmentally friendly than using fossil fuels. However, little is known about their deployment in the actual environment and its impact on marine life acoustics. The potential impact of Underwater Radiated Noise (URN) from marine propellers has recently been considered by Frisk (2012) concluding an increasing trend in underwater ambient noise due to shipping activities. Investigations and measurements of URN of marine propellers as well as tidal turbines has also been investigated (Aktas et al, 2016a; Aktas et al, 2016b; Shi et al, 2016) although none of these studies here considered the noise impact of tidal current turbines on the marine environment.

Marine animals communicate using sound with different pitches and frequencies. This communication is essential when migrating, hunting for food and mating. When this channel of communication is interrupted, there will be impact on the behaviours and existence of the animals (Richardson et al, 2013; White \& Pace, 2010). There are five types of noise, ambient and radiated noise being two of them (Wenz, 1972). While ambient noise is the baseline noise of the underwater environment that comes from different sources, radiated noise is defined as the unwanted noise produced by ma-made marine systems such as ships (Ross, 1976). Wenz (1972) revealed that shipping noise dominated the low frequency range of the ambient noise. 
Research into Underwater Radiated Noise (URN) from the marine industries is abundant, ranging from propellers and shipping noise (Aktas et al, 2016b; Hildebrand, 2009; Williams et al, 2015) to URN from offshore wind turbines (Madsen et al, 2006) and piling noise (Spiga, 2016; Thomsen et al, 2012). Additionally, research in the marine science field on the impact of radiated noise from marine activities on marine life is also explored (Brownell et al, 2008; Hawkins et al, 2008; Spiga et al, 2012). Marine noise pollution is recognized as a significant threat to marine diversity and initiatives need to be taken to study the URN characteristics from renewable energy devices (Simmonds et al, 2014) before they are extensively deployed.

In terms of URN from marine activities in general, different research projects were conducted such as the SILENV (Ships oriented Innovative soLutions to rEduce Noise and Vibrations). The AQUO (Achieve Quieter Oceans) and SONIC (Suppression Of underwater Noise Induced by Cavitation) exploring underwater cavitation noise related issue. International organizations and committees such as the International Maritime Organization (IMO) and the Marine Environmental Protection Committee (MEPC) are looking into URN from commercial shipping to help develop potential guidelines and regulations (SONIC, 2012).

As the tidal energy industry is rapidly growing, with increasing deployment of devices whether for research or grid-connected, the operation of tidal turbines will contribute to the generation of URN. With regard to marine current turbines, work on the radiated noise level measurement from model testing has been conducted (Shi et al, 2016; Wang et al, 2004; Wang et al, 2007) as well as investigations into the influence of turbulence on noise generation using numerical approaches (Lloyd et al, 2011; 2013; 2014). The ÆMORE project (Walsh et al, 2015) proposed using URN as a monitoring technique to detect sound anomalies that can be contributed by impending component failure of the converters. From the ecological and biological approach, various studies have been conducted to understand the impact of URN from tidal energy converters on marine animal behaviour and health (Halvorsen et al, 2011; Pine et al, 2016; Spiga, 2014; Wilson et al, 2014).

Not only is noise radiated from the operation of marine machinery, but their installation, maintenance and decommissioning would also cause disturbance to the ambient noise level of the marine environment. Parallels can be drawn with the offshore wind industry, where URN from the installation of wind farms is found to interfere with marine mammals' acoustic range which may cause behavioural disturbance (Bailey et al, 2010; Madsen et al, 2006). Similarly, 
tidal turbine operations also generate URN from the turbulence in the flow due to the turbine and installation system, generator noise, and to on-shore power transmission. URN from both non-turbine operation as well as turbine operations will have an impact on the natural marine acoustic environment in which they are deployed. URN from non-turbine operation activities could be detrimental even though it occurs over a shorter period of time, however URN due to turbine operations affects the marine habitat and ecology over the long term (Bevelhimer et al, 2016).

In addition, tidal turbine operations are susceptible to cavitation which has been observed in two independent studies using different turbine designs and test facilities (Bahaj et al, 2007; Wang et al, 2007). Both papers studied the different types of cavitation that occurred during the test; tip vortex, sheet and cloud cavitation were observed in Emerson Cavitation Tunnel tests conducted where noise measurements were also obtained (Wang et al, 2007). Cavitation in tidal turbine operations will only increase the URN and cause more disturbance to the marine environment. To date, there is no complete full scale tidal turbine URN data from tidal turbines available in the literature.

When investigating cavitation on a scaled model, cavitation may not accurately predict the cavitation behaviour at full scale level due to scale effects (Ross, 1976). Moreover, Molland et al (2004) found numerical calculation under-predicts cavitation observed on laboratory scaled models. Another key concern is the difference in Reynold's Number between a scaled model and full scale prototype. It is therefore important to set the scaled Reynold's Number as high as possible to reduce weighty discrepancy in the cavitation observations as well as the Reynold's Number scaled effect. The scaled effect is understood to be negligible when the value is above $10^{6}$ (Wang et al, 2007). Taking consideration of blockage effect and other limitation, the Reynold's Number was set between $1-5 \times 10^{6}$ in the test.

This paper aims to look into the URN from Hydro-Spinna turbine operation predicted from a model scale measurements and offer an indication on how the full scale turbine model operates. Due to its unique design, the Hydro-Spinna turbine is believed to produce a different acoustic profile compared to conventional horizontal axis tidal current turbines. The model measurements were analysed and extrapolated into full scale noise predictions using the scaling law recommended by the ITTC (ITTC, 1987) for marine propellers as at present there is no 
accepted method for scaling for tidal turbines. The established ITTC scaling law was employed due to the similarity between propellers and turbines.

The extrapolations will involve noise data measured at different turbine cavitation numbers and observations. The noise was measured using a miniature hydrophone at different Tip Speed Ratio (TSR) and different cavitation numbers. Cavitation observations were also conducted by using two high speed cameras. The results were then extrapolated using the previously mentioned scaling law for propellers to forecast the noise generated from three full scales diameters turbine. The full scale noise predictions are plotted against the International Council for the Exploration of the Sea (ICES) fish reaction threshold (Mitson, 1995). The ICES criteria is imposed on the noise reduction criteria of fishing research vessels conducting fisheries related research. Therefore, the comparison of the full scale noise prediction with the ICES level is purely on comparison basis.

\section{Turbine Design and Performance}

\subsection{Turbine Design and Model}

The Hydro-Spinna is a turbine with three helicoidal blades. The blades have a cardioid shaped profile that is spiralled around the central hub of the turbine, as shown in Fig. 1. The turbine model that was tested has blades that have a cardioid shaped leading edge and circular trailing edge. 

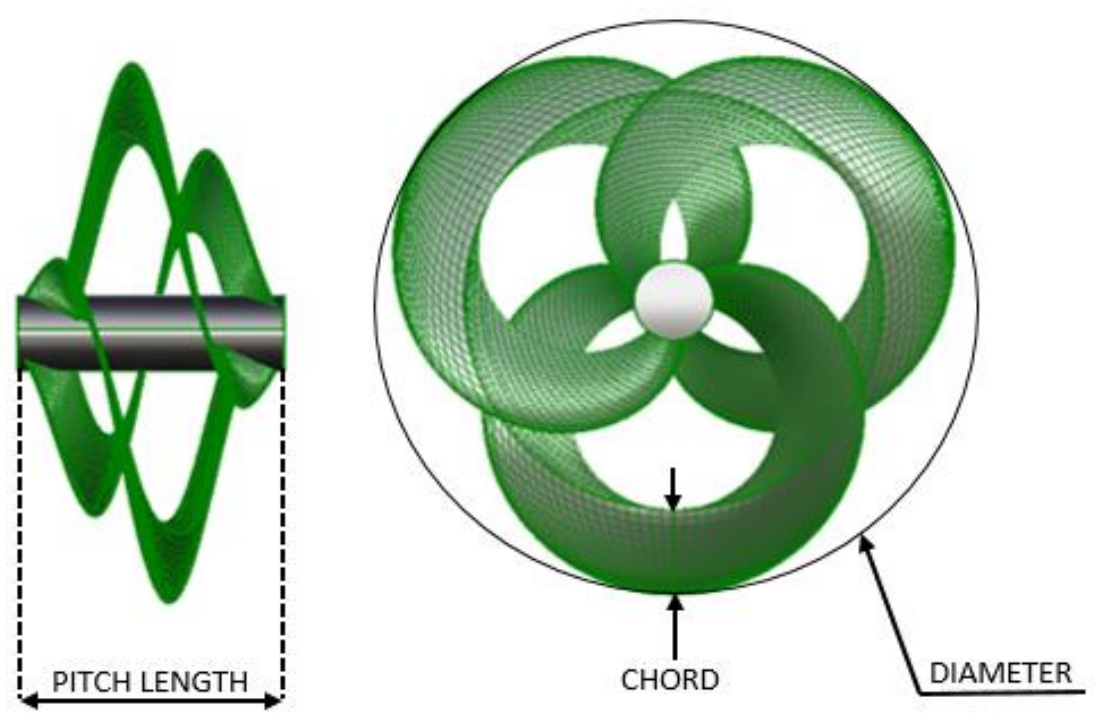

Fig. 1: The $280 \mathrm{~mm}$ diameter Hydro-Spinna model with NACA 0006 blade profile and blade parameters highlighted.

The model blade cross section is shaped as an aerodynamic profile of NACA 0006. The pitch length $(p)$ of the model turbine is the distance between the upstream and downstream ends in the axis parallel to the flow. The diameter $(D)$ is the diameter of the turbine swept area which is $280 \mathrm{~mm}$ for this turbine model. The model has a pitch length of $112 \mathrm{~mm}$ giving a final pitch to diameter ratio of 0.43 . The turbine specification is listed in Table 1 to provide details of the design.

Table 1: The chord length and pitch angle distribution with radius of the HS280 HydroSpinna brass model

\begin{tabular}{c|c|c}
$\mathbf{r} / \mathbf{R}$ & $\mathbf{C} / \mathbf{R}$ & Pitch Angle, $\left.\boldsymbol{\beta} \mathbf{(}^{\mathbf{0}}\right)$ \\
\hline 0.1000 & 0.2007 & 57.86 \\
0.2064 & 0.2400 & 37.63 \\
0.3390 & 0.3080 & 25.15 \\
0.4860 & 0.3970 & 18.13 \\
0.6100 & 0.5055 & 14.62 \\
0.7143 & 0.8254 & 12.56 \\
0.8302 & 0.7180 & 10.85 \\
0.9128 & 0.5555 & 9.89 \\
1.0000 & 0.0000 & 9.05
\end{tabular}




\subsection{Hydro-Spinna Performance}

The radiated noise level was measured as part of a systematic cavitation test of the HydroSpinna turbine. Power and thrust coefficients were not measured during this round of tests as the performance of the Hydro-Spinna turbine had been conducted in an earlier test (Rosli et al, 2015). For the power and thrust performance investigation, the turbine was driven by an H33 Kempf \& Remmers dynamometer. The dynamometer was used to regulate the turbine rotational speed while also measuring the torque and thrust of the turbine. The dynamometer has a maximum thrust of $2943 \mathrm{~N}$, maximum torque of $147 \mathrm{Nm}$ and maximum rotational speed of 4000 revolution per minute (rpm).

The Power Coefficient $\left(\mathrm{C}_{\mathrm{P}}\right)$, Thrust Coefficient $\left(\mathrm{C}_{\mathrm{T}}\right)$ and TSR are defined in Equations $1-3$ below. The experimental power and thrust coefficient of the Hydro-Spinna turbine is presented in Fig. 2. The investigation was conducted for a tunnel velocity of $3 \mathrm{~m} / \mathrm{s}$ and tunnel pressure of $850 \mathrm{~mm} \mathrm{Hg}$ which is the normal atmospheric pressure. The power coefficient peaks at a value of 0.38 at optimal TSR $=3$ where the thrust coefficient is $C_{t} / 10=0.085$, while the thrust coefficient peaks at $T S R=1$, at a value of $\mathrm{C}_{\mathrm{t}} / 10=0.12$. The thrust loading trend on the HydroSpinna is unique as it peaks at low TSR and then decreases as TSR increased. At the starting condition i.e. $T S R=0$, the thrust coefficient is approximately $\mathrm{C}_{t} / 10=0.09$ and then increased until it peaks at $T S R=1$. This trend will affect the cavitation behaviour of the Hydro-Spinna turbine which will be discussed in detail in Section 4.

$$
\begin{aligned}
& C_{P}=\frac{Q \Omega}{0.5 \rho A U^{3}} \\
& C_{T}=\frac{T}{0.5 \rho A U^{2}} \\
& T S R=\frac{\Omega R}{U}
\end{aligned}
$$

where $Q$ is the torque, $T$ is the thrust, $\Omega$ is the angular velocity of the turbine, $\rho$ is the density of the fluid, $A$ is the swept area of the turbine, and $U$ is the flow velocity. 


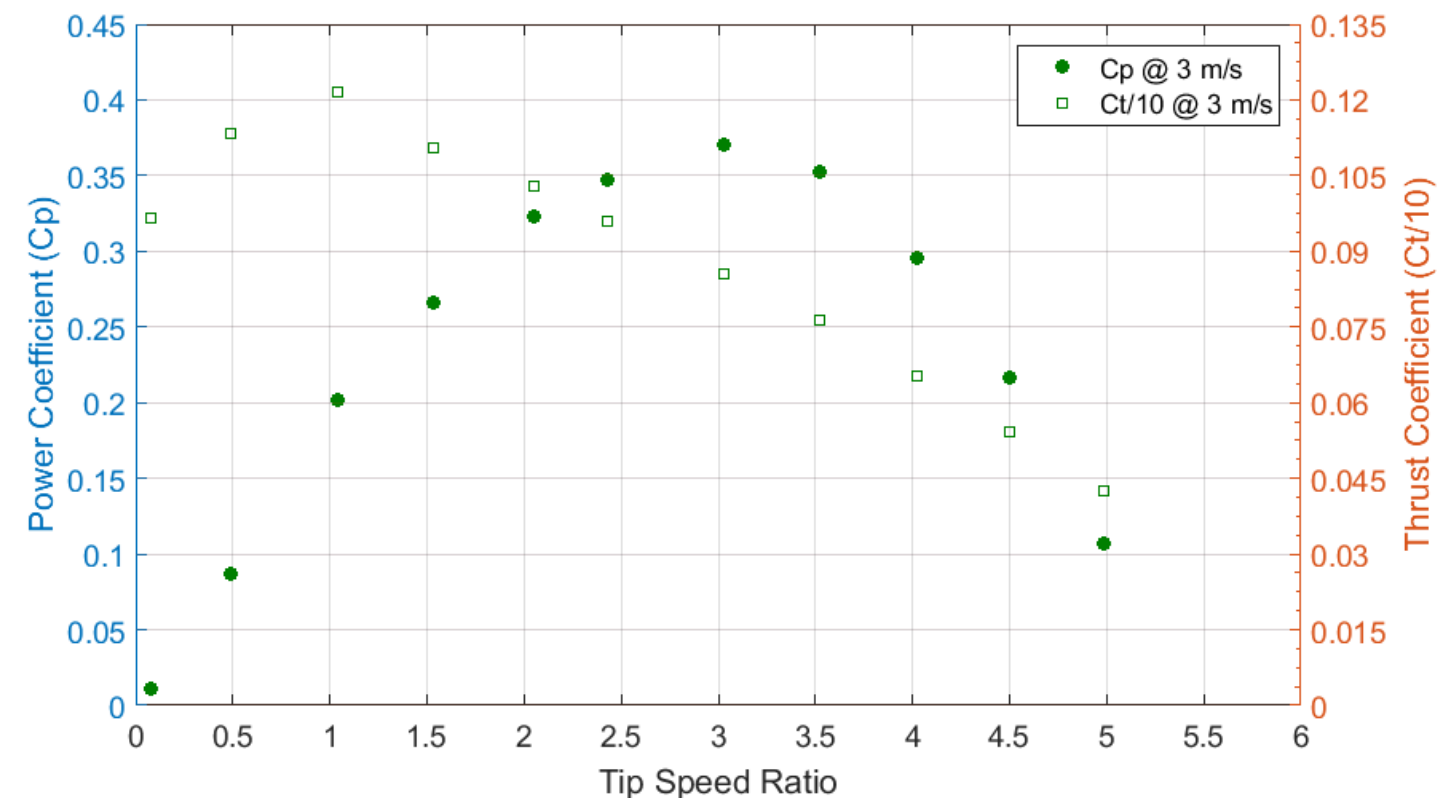

Fig. 2: The power and thrust coefficient of the Hydro-Spinna turbine

\subsection{Numerical Model}

In addition to the experimental data, a numerical study was developed to support the results. The numerical model employed Reynolds-Averaged Navier Stokes Equations (RANS) and Menter SST $k$ - $\omega$ turbulence model for the theoretical analysis. The domain size used in this investigation was set to the dimensions of the ECT. The numerical domain has a sub-domain which is the rotating domain containing the turbine as shown in Fig. 3. The rotating domain modelled the rotation of the turbine using the Moving Reference Frame (MRF) technique. The rotating domain had three times the radius of the turbine, with the upstream and downstream boundaries set at 2D distance from the turbine centre. The upstream velocity was kept the same as the experiment at $3 \mathrm{~m} / \mathrm{s}$ while the domain wall was set to no-slip condition.

The numerical results obtained was found to agree with the experimental data as shown in Fig. 4, with the power coefficient results closely corresponding to each other. However, the numerical model underpredicted the thrust coefficient by $8 \%$ at the peak TSR. Nonetheless, the numerical findings have further justified the experimental investigation both in the power and thrust coefficient of the Hydro-Spinna turbine. 


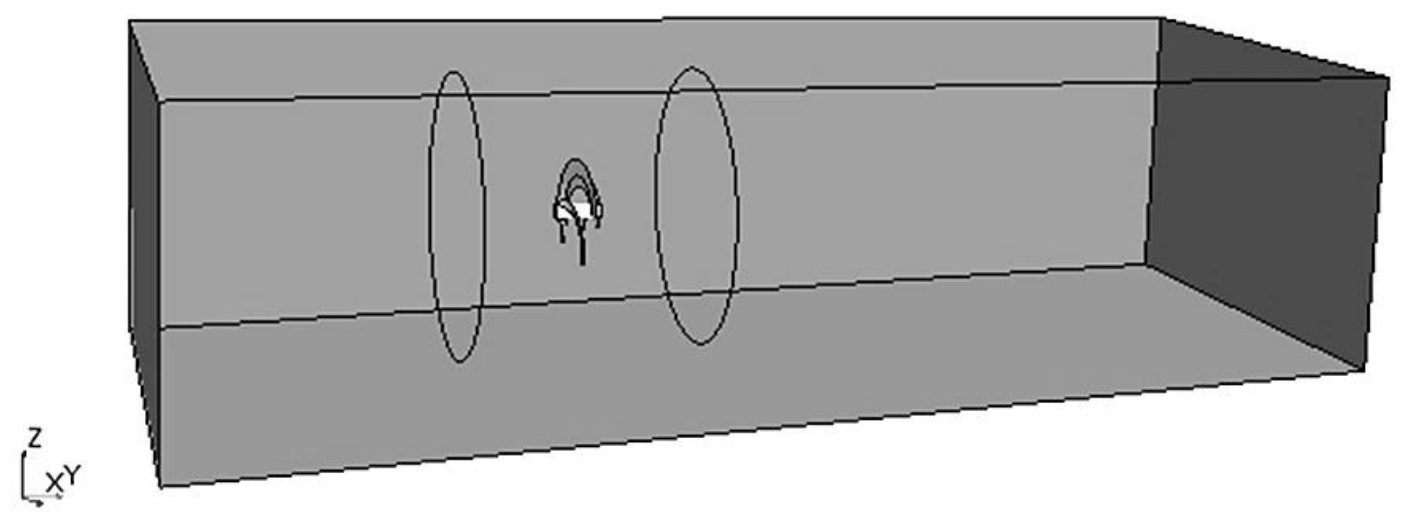

Fig. 3: Numerical model with the ECT dimensions as its main domain and a rotating cylindrical sub-domain

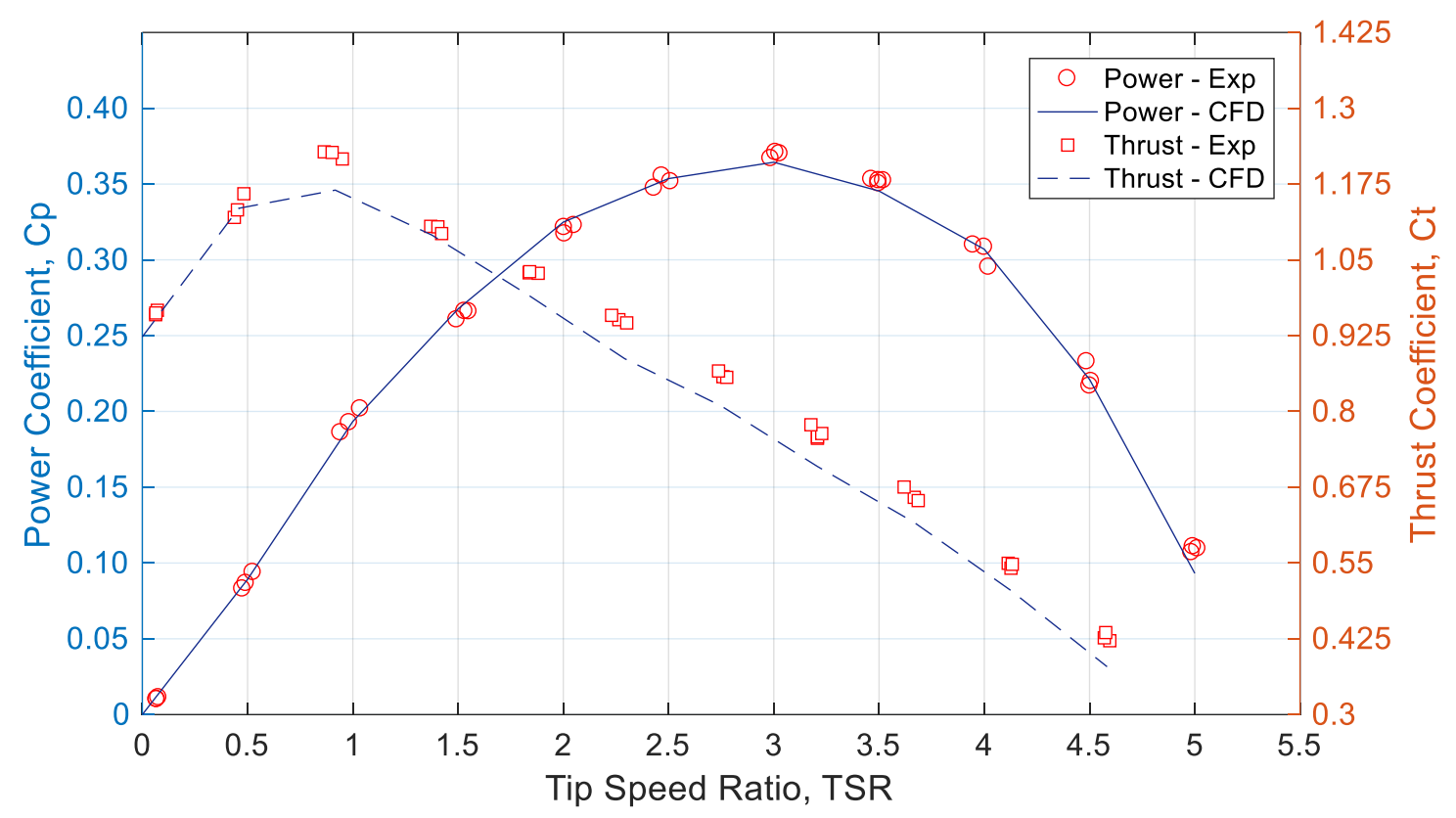

Fig. 4: The experimental and numerical power and thrust coefficient of the Hydro-Spinna turbine 


\section{Test Facilities and Procedure}

\subsection{Facilities and Equipment}

The noise measurement and cavitation observation tests were conducted in the Emerson Cavitation Tunnel at Newcastle University. The tunnel has the following dimensions, while its further details can be found in (Atlar, 2011):

\section{$\underline{\text { Test Section }}$}

Dimensions

Area

Contraction Ratio

Maximum Velocity
$3.1 \mathrm{~m} \times 1.21 \mathrm{~m} \times 0.8 \mathrm{~m}(\mathrm{~L} \times \mathrm{B} \times \mathrm{H})$

$1.008 \mathrm{~m}^{2}$

4.271

$8 \mathrm{~m} / \mathrm{s}$

As mentioned previously, the Hydro-Spinna turbine was driven by a H33 Kempf \& Remmers that controls the rotational speed of the turbine to obtain the power and thrust data for a full range of turbine operational speeds. Concurrently, the noise level was measured using a Bruel and Kjaer (B\&K) Type 8103 miniature hydrophone positioned inside the tunnel $0.38 \mathrm{~m}$ away from the centre of the turbine as shown Fig. 5. A dedicated (B\&K) PULSE Type 3023 system with a 6/1 LAN interface data acquisition system was used to process the noise results both in $1 \mathrm{~Hz}$ and 1/3 Octave Band. 


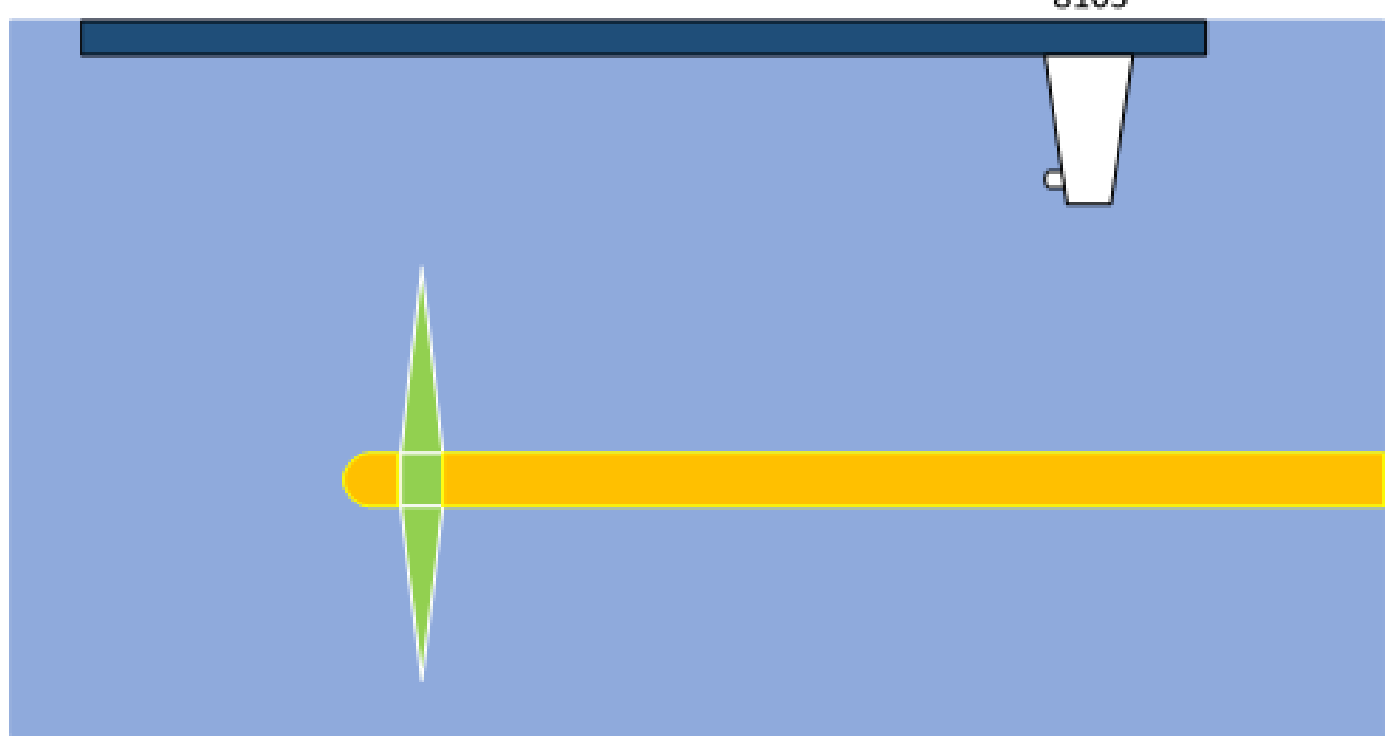

Fig. 5: An overview of the turbine in the Emerson Cavitation Tunnel and location of the B\&K miniature hydrophone

Two high speed cameras, Nano Sense MK II and III, were also used during the tests to provide visual observation of the turbine operation and any cavitation inception. The cameras were triggered by the tunnel rpm counter and synchronized with a continuous Plasma Lite 400 light to illuminate the tunnel during recording. The cameras set up is shown in Fig. 6. 


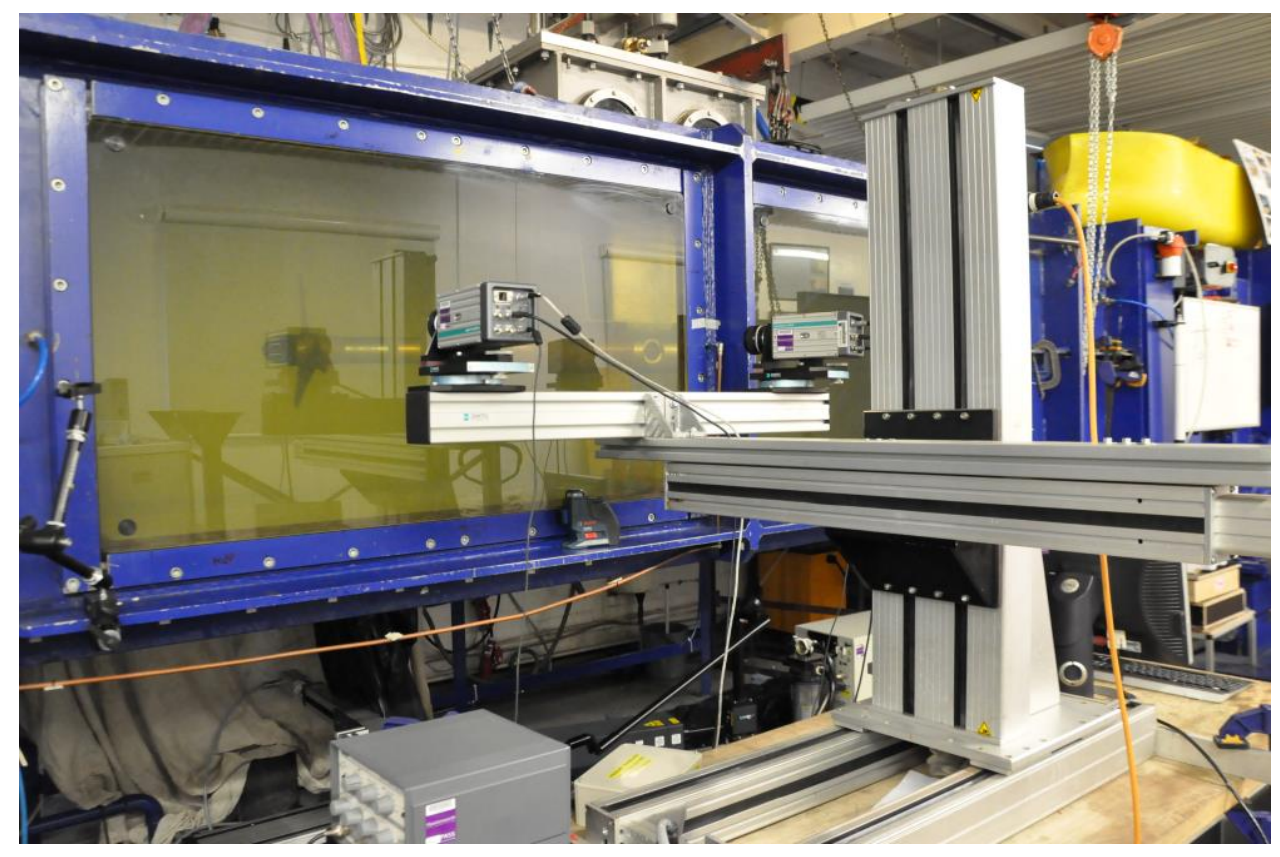

Fig. 6: High speed cameras set up for cavitation observation

\subsection{Test Matrix}

The noise measurement tests were conducted for the full range of TSR at different pressure levels, which are the normal tunnel pressures of $850 \mathrm{~mm} \mathrm{Hg}$, medium and high vacuum tunnel pressures of $550 \mathrm{~mm}$ and $250 \mathrm{~mm} \mathrm{Hg}$ respectively. The test matrix is described in Table 2.

Table 2: Test matrix for the noise measurement and cavitation tests

\begin{tabular}{|c|c|c|c|c|}
\hline Test & Pressure & Tunnel Velocity & $T S R$ Range & $\begin{array}{c}\text { Cavitation } \\
\sigma_{0.7 \mathrm{R}}\end{array}$ \\
\hline \multirow{6}{*}{1} & \multirow{6}{*}{$\begin{array}{l}\text { Normal Tunnel Pressure } \\
\text { i.e. Atmospheric Pressure } \\
\qquad(850 \mathrm{~mm} \mathrm{Hg})\end{array}$} & \multirow{9}{*}{$3 \mathrm{~m} / \mathrm{s}$} & 0 (Start) & 24.3 \\
\hline & & & 1 & 16.2 \\
\hline & & & 2 & 8.02 \\
\hline & & & 3 & 4.51 \\
\hline & & & 4 & 2.67 \\
\hline & & & 5 & 1.80 \\
\hline \multirow{3}{*}{2} & \multirow{3}{*}{$\begin{array}{l}\text { Medium vacuum pressure } \\
\qquad(550 \mathrm{~mm} \mathrm{Hg})\end{array}$} & & 0 (Start) & 15.6 \\
\hline & & & 1 & 10.2 \\
\hline & & & 2 & 5.15 \\
\hline
\end{tabular}




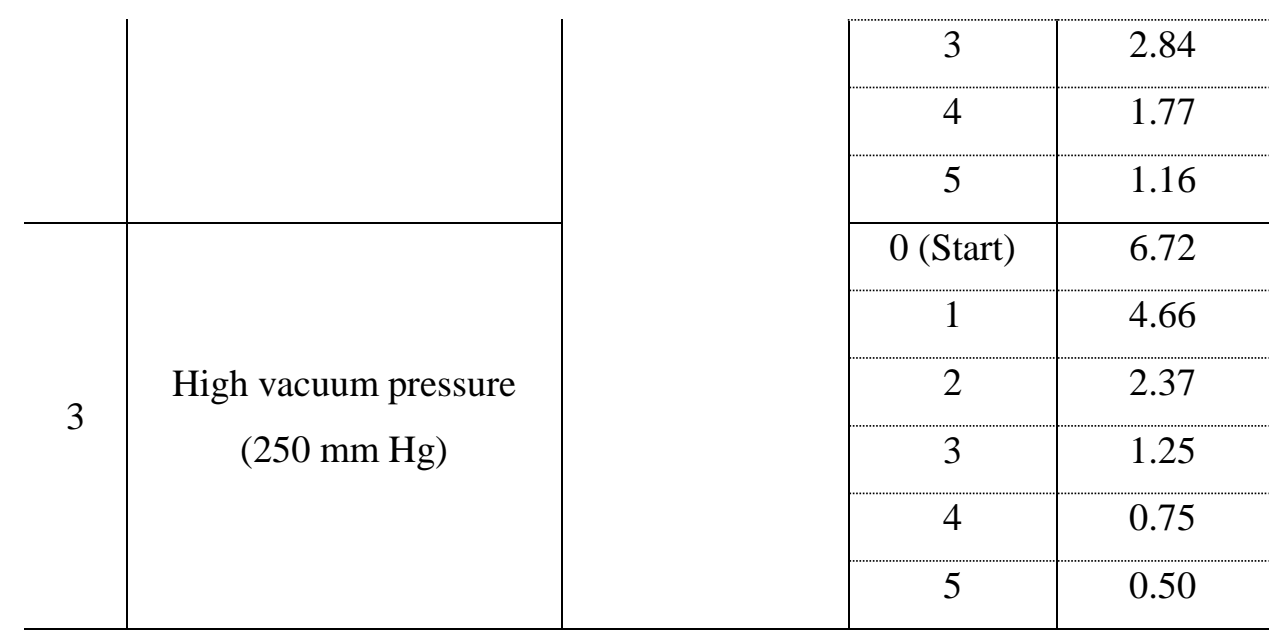

A total of 18 tests were conducted where for each pressure, measurements and observations were made for a complete range of the Hydro-Spinna operational TSRs each with its cavitation number also calculated.

\section{Cavitation Observations}

Tidal turbines are prone to cavitation at certain operating conditions specifically at high flow velocity and turbine rotational speed. Cavitation is undesirable in turbine operation as it can erode the turbine blades and disrupt the flow of the fluid as well as generating noise. Cavitation occurs primarily when the local pressure around the turbine, $P_{L}$, is lower than the vapour pressure of the flow, $P_{V}$ assumed to be $1200 \mathrm{~Pa}$. Cavitation number is used to describe the resistance to cavitation, hence when the cavitation number is low, the turbine is more susceptible to cavitation. The cavitation number for the Hydro-Spinna cavitation test was taken at the blade section where its radius is $0.7 R$, where the radius is 0.7 times the radius, $R$, of the turbine as defined in Equation 4.

$$
\sigma_{0.7 R}=\frac{P_{T}-\rho g(0.7 R)-P_{V}}{0.5 \rho W_{0.7}{ }^{2}}
$$

Equation 4

where $\boldsymbol{W}$ is the resultant flow velocity at $0.7 \mathrm{R}$ defined as $\boldsymbol{W}_{\mathbf{0 . 7}}=\sqrt{(\boldsymbol{\Omega} \times \mathbf{0 . 7 R})^{\mathbf{2}}+\boldsymbol{U}_{\boldsymbol{o}}^{\mathbf{2}}}$, the term $P_{T}-\rho g(0.7 R)$ is the static pressure above the location $0.7 R, P_{T}$ is the tunnel pressure, $\rho$ is the water density, $U_{O}$ is the flow velocity, and $\Omega$ is the rotational velocity of the turbine. 


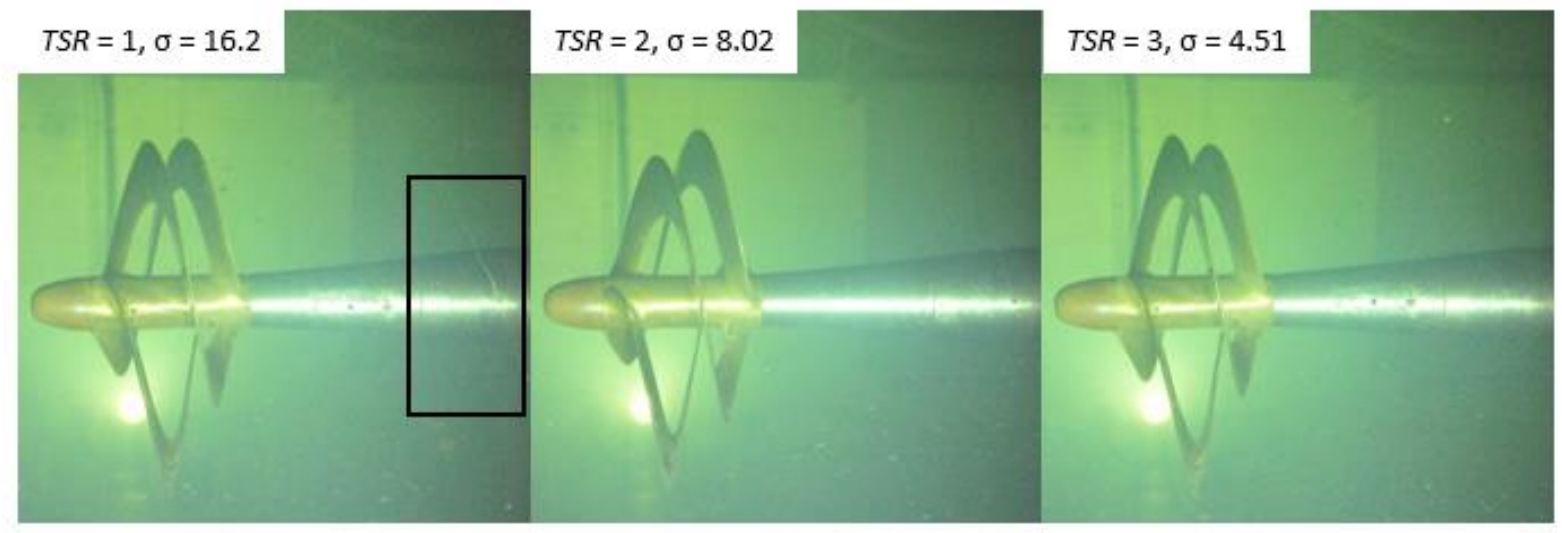

Fig. 7: Cavitation observation at normal tunnel pressure

At normal tunnel pressure, it was observed that there was a weak intermittent tip vortex cavitation at $T S R=1$ where coincidentally the thrust coefficient on the turbine is the highest. This was the only condition where cavitation was observed, as shown in Fig. 7. Most importantly, the Hydro-Spinna turbine operates cavitation free at its optimal TSR $=3$. As the pressure is reduced to medium vacuum pressure, a steadier tip vortex cavitation was observed at $T S R=1$, and a weak intermittent tip vortex was also observed at $T S R=2$, as shown in Fig. 8. As with the normal tunnel pressure case, the tip vortex cavitation tends to dissipate with increasing TSR where at optimal operation speed, no cavitation was observed.

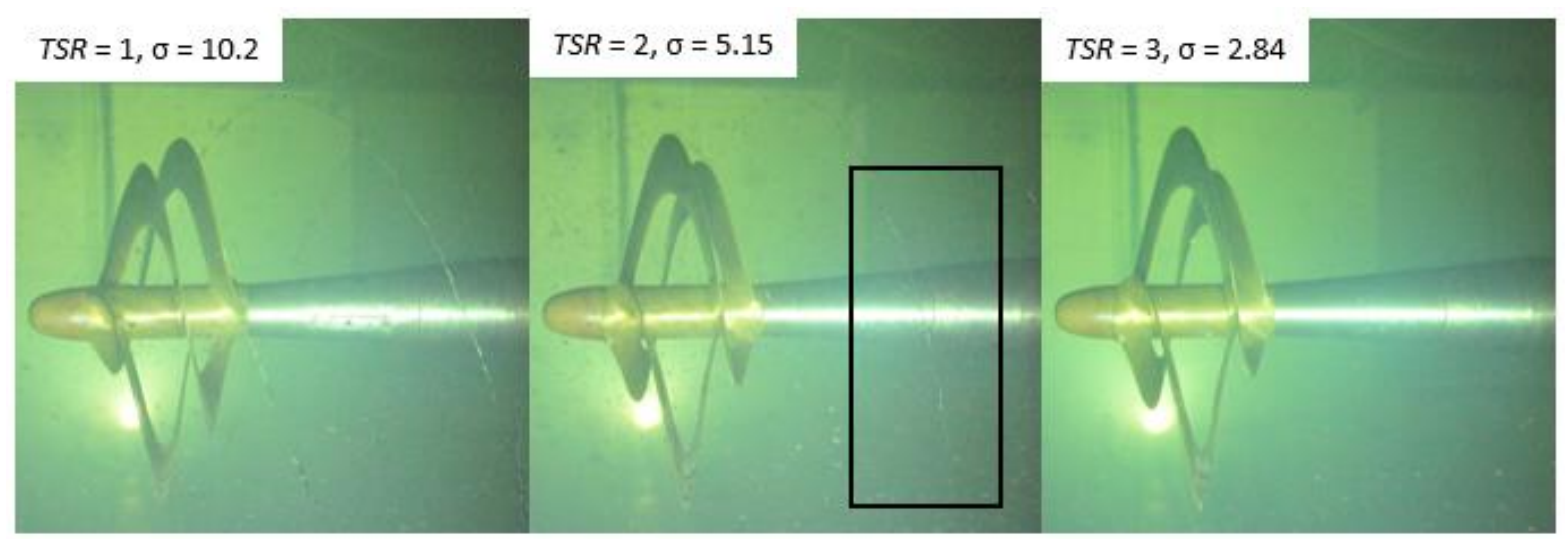

Fig. 8: Cavitation observation at medium vacuum pressure 
At high vacuum pressure, strong and steady tip vortices were observed at $T S R=1$ and 2 . Two sets of tip vortex cavitation were observed where the second set appeared to begin from the middle section of the turbine. The smaller tip vortex seemed to converge towards the shaft rather than expanding as it dissipated downstream, as shown in Fig. 9. The outer tip vortex, originating from the outer leading edge of the turbine expanded as it dissipated downstream. At the optimal TSR, very weak discontinuous tip vortex cavitation was observed. As with the other two cases, the cavitation dissipated with increasing TSR.

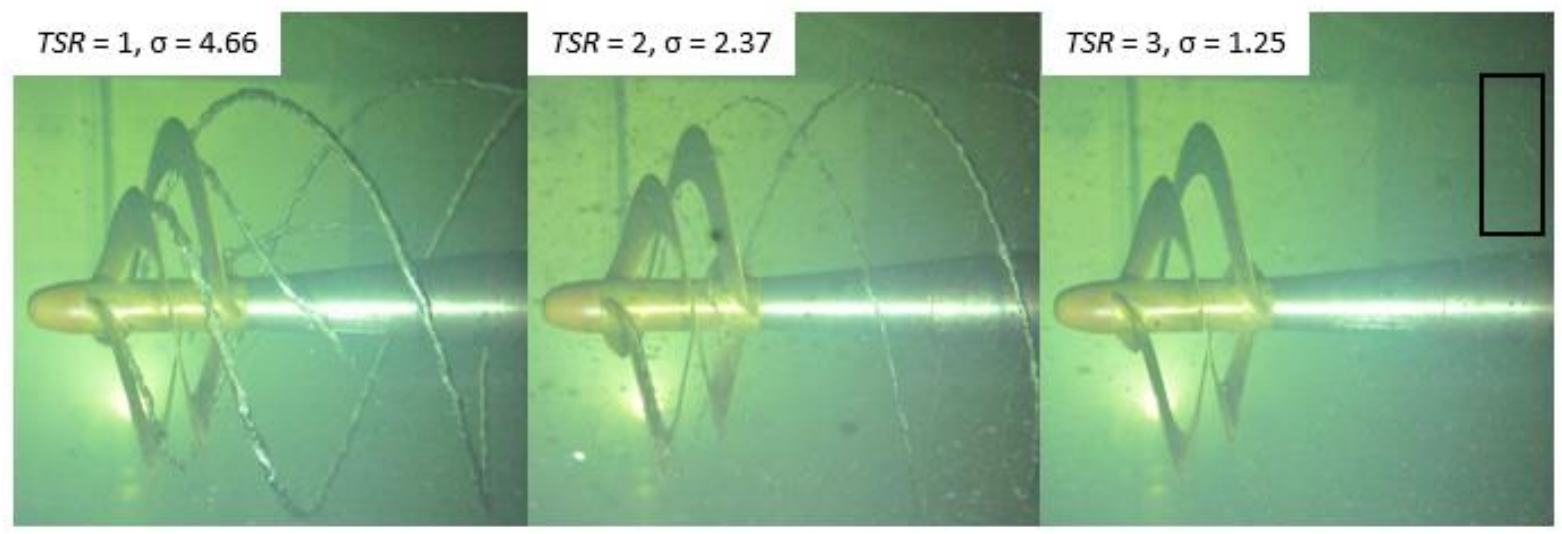

Fig. 9: Cavitation observation at high vacuum pressure

Typically, with conventional horizontal axis tidal turbine, as TSR increases, the thrust coefficient also increases corresponding to a decrease in the cavitation number. Generally, cavitation is observed to occur as the cavitation number decreases (Bahaj et al, 2007; Wang et al, 2007). Therefore, it is well understood that cavitation will occur with lower cavitation number. However, the same cannot be concluded for the Hydro-Spinna turbine as, even though the cavitation number deceases with increasing TSR, the thrust coefficient decreases instead. For the Hydro-Spinna turbine, the relationship between thrust coefficient and cavitation number is unusual to the norm. Therefore, in this case, low cavitation number does not dictate that cavitation will occur as it does not represent the increase in thrust coefficient anymore. In fact, the cavitation observations were observed to be closely correlated to the thrust coefficient trend of the turbine where the turbine only cavitated at high thrust coefficient conditions. Fig. 10 indicated the cavitation observed at different tunnel pressure against the thrust coefficient. At all pressure, cavitation was observed at $T S R=1$ where the thrust coefficient is the maximum. Table 3 presents a summary of the cavitation observation for more clarity. 


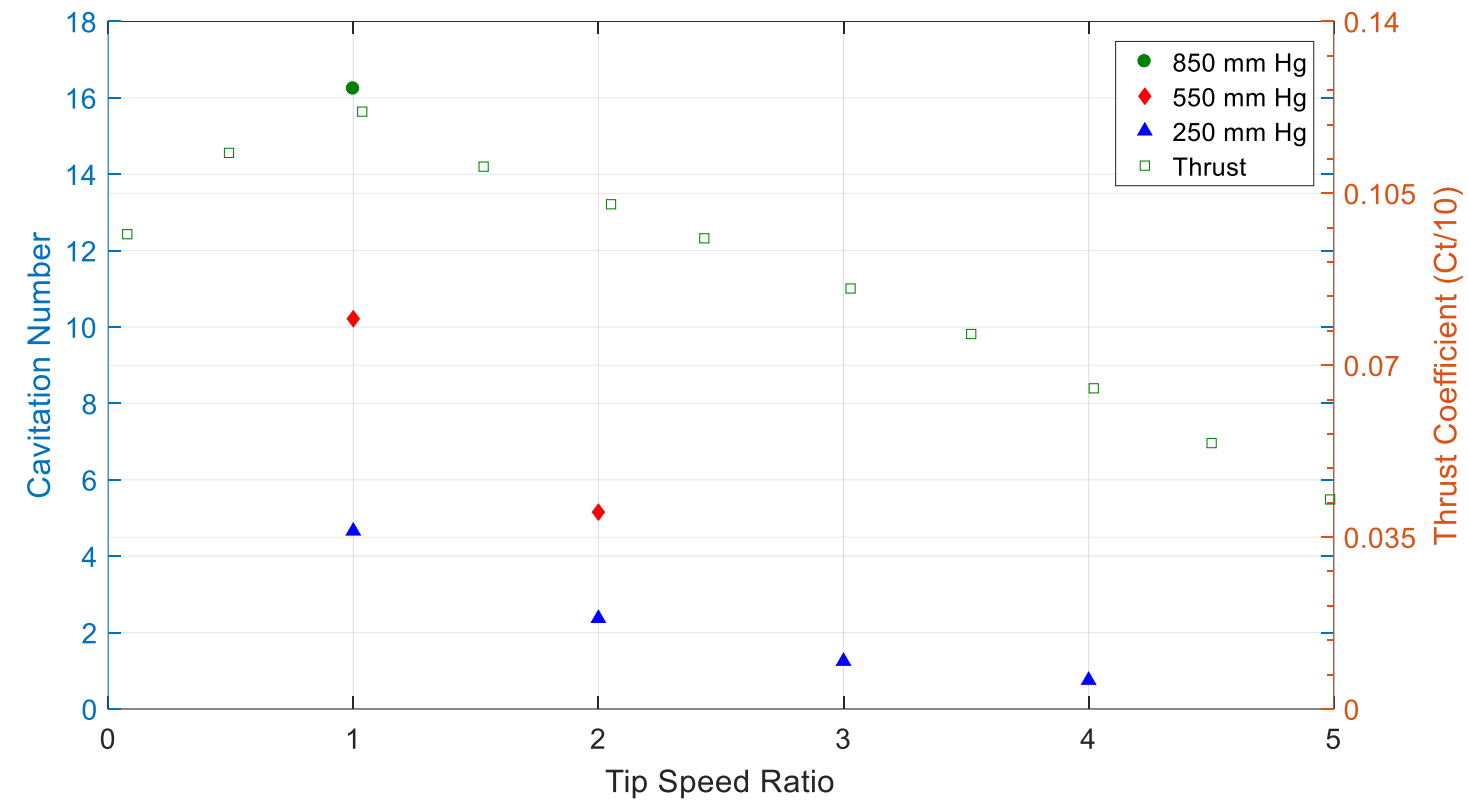

Fig. 10: Comparison of cavitation observed at different tunnel pressure to the thrust coefficient

Table 3: A summary of the cavitation observations on the Hydro-Spinna turbine at different tunnel pressure.

\begin{tabular}{|c|c|c|c|c|}
\hline Test & Pressure & $T S R$ & $\begin{array}{c}\text { Cavitation } \\
\sigma_{0.7 \mathrm{R}}\end{array}$ & Observation \\
\hline \multirow{6}{*}{1} & \multirow{6}{*}{$\begin{array}{l}\text { Normal Tunnel } \\
\text { Atmospheric } \\
\text { Pressure } \\
(850 \mathrm{~mm} \mathrm{Hg})\end{array}$} & 0 & 24.3 & No \\
\hline & & 1 & 16.2 & Yes \\
\hline & & 2 & 8.02 & No \\
\hline & & 3 & 4.51 & No \\
\hline & & 4 & 2.67 & No \\
\hline & & 5 & 1.80 & No \\
\hline \multirow{6}{*}{2} & \multirow{6}{*}{$\begin{array}{c}\text { Medium vacuum } \\
\text { pressure } \\
(550 \mathrm{~mm} \mathrm{Hg})\end{array}$} & 0 & 15.6 & No \\
\hline & & 1 & 10.2 & Yes \\
\hline & & 2 & 5.15 & Yes \\
\hline & & 3 & 2.84 & No \\
\hline & & 4 & 1.77 & No \\
\hline & & 5 & 1.16 & No \\
\hline \multirow{3}{*}{3} & \multirow{3}{*}{$\begin{array}{l}\text { High vacuum } \\
\text { pressure } \\
(250 \mathrm{~mm} \mathrm{Hg})\end{array}$} & 0 & 6.72 & No \\
\hline & & 1 & 4.66 & Yes \\
\hline & & 2 & 2.37 & Yes \\
\hline
\end{tabular}




\begin{tabular}{l|c|c|c}
\hline \multirow{4}{*}{} & 3 & 1.25 & Yes \\
\cline { 2 - 4 } & 4 & 0.75 & No \\
& 5 & 0.50 & No \\
\hline
\end{tabular}

\section{Analysis and Presentation of Noise Model Results}

The noise data was processed using the PULSE lab-shop using Constant Percentage Bandwidth (CPB) and Fast Fourier Transform (FFT) analysers in the dedicated software. The measurements were made for an average of 50 seconds in $1 / 3$ Octave band level for $20 \mathrm{~Hz}$ to $20 \mathrm{kHz}$ and $1 \mathrm{~Hz}$ band levels. The raw output noise measurements were corrected to $1 \mathrm{~Hz}$ bandwidth and $1 \mathrm{~m}$ source level as is the common practice with propeller noise measurements (ITTC, 1987). The correction equation for the Sound Pressure Level (SPL) to an equivalent 1 $\mathrm{Hz}$ bandwidth and standard distance of measurements of $1 \mathrm{~m}$ are given respectively in Equations 5 and 6 below:

$$
\begin{array}{ll}
\mathrm{SPL}_{1}=\mathrm{SPL}_{m}-10 \log \Delta \boldsymbol{f} & \text { Equation } 5 \\
\mathrm{SPL}=\mathrm{SPL}_{1}+2 \operatorname{2og}(\boldsymbol{r}) & \text { Equation } 6
\end{array}
$$

where SPL is the Sound Pressure Level in $1 \mathrm{~Hz}$ band in $\mathrm{dB}$ relative to $1 \mu \mathrm{Pa}$ at $1 \mathrm{~m}, \mathrm{r}$ is the distance of the location of the hydrophone from the turbine centreline, SPL 1 is the Sound Pressure Level in $1 \mathrm{~Hz}$ band in $\mathrm{dB}$ relative to $1 \mu \mathrm{Pa}$, SPL $m$ is the Sound Pressure Level in 1/3 Octave band in $\mathrm{dB}$ relative to $1 \mu P a$, and $\Delta f$ is the frequency bandwidth for the $1 / 3$ Octave band for each centre frequency.

The cavitation noise experiments need utmost attention during the execution of the test as there are a number of factors that require correction or disposal of the data. The most influential factor is the background noise of the ECT. Background noise was determined during the investigation at the exact operational conditions of the cavitation tests, where a dummy boss replaces the turbine. The background noise needs to be removed if the difference between the background levels and the turbine cavitation noise level is less than $3 \mathrm{~dB}$ and a correction is applied if the difference is between 3 to $10 \mathrm{~dB}$ as described in Equation 7 (ANSI, 2009; Bertschneider et al, 2012). The major noise sources contributing to the background noise are 
turbulent boundary layer noise, turbine noise, the dynamometer and motor noise. The background noise corrections were applied to the Hydro-Spinna noise measurements to eliminate the impact of background noise as well as the self-noise of the hydrophone due to the water flow.

$$
\mathrm{SPL}_{N}=10 \log \left[10^{\left(\mathrm{SPL}_{T} / 10\right)}-10^{\left(\mathrm{SPL}_{B} / 10\right)}\right]
$$

where SPL $N$ is the net Sound Pressure Level, SPL $T$ is the total Sound Pressure Level and SPL $B$ is the background noise Sound Pressure Level.

As cavitation tunnels are closed circuits with a chamber type measuring section, they are highly reverberant environments. Therefore, the influence of the testing environment on the noise transfer function needs to be determined in order to properly relate measured SPL to source level at a normalized distance of $1 \mathrm{~m}$ (Park et al, 2009). Such correction is determined by substituting a calibrated noise source for the turbine. The hydrophone measurement and the calibrated source levels are then compared in order to determine the transfer function of the sound within the testing section. Transfer functions has been determined for ECT but have not been applied for the results of this study. 


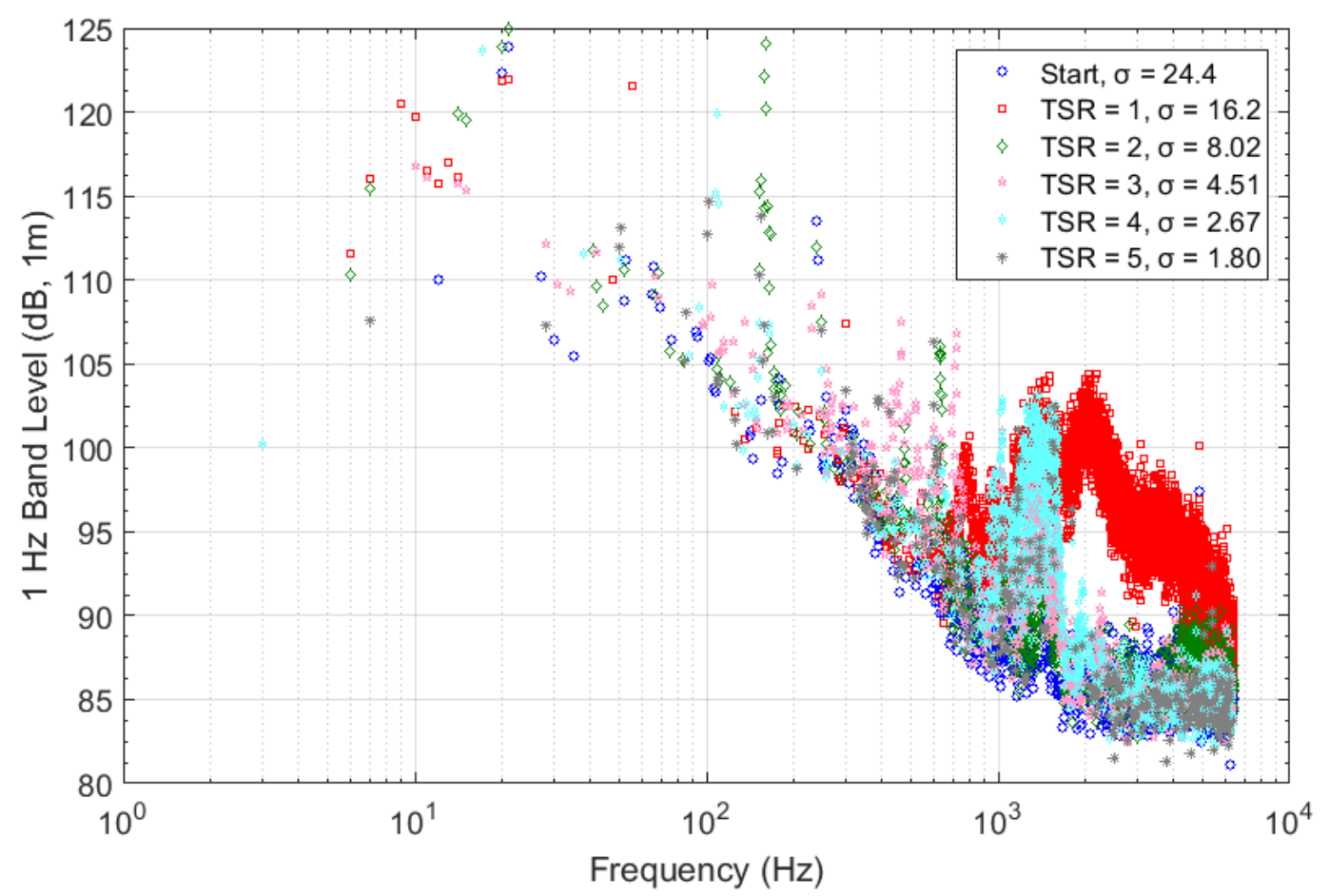

Fig. 11: Net $1 \mathrm{~Hz}$ Band Level at all Tip Speed Ratio (TSR) at tunnel pressure

Both the $1 \mathrm{~Hz}$ band and the 1/3 Octave band level were corrected and analysed as shown respectively in Fig. 11 and Fig. 12. Background noise was eliminated in both cases. The $1 \mathrm{~Hz}$ band shows a significant increase in radiated noise level at $T S R=1$ where the thrust coefficient is the highest. The 1/3 Octave yields radiated noise data at $T S R=1$ and 2 only. The increase in noise measured in the $1 \mathrm{~Hz}$ band data at $T S R=1$ was also observed in the $1 / 3$ Octave band data. 


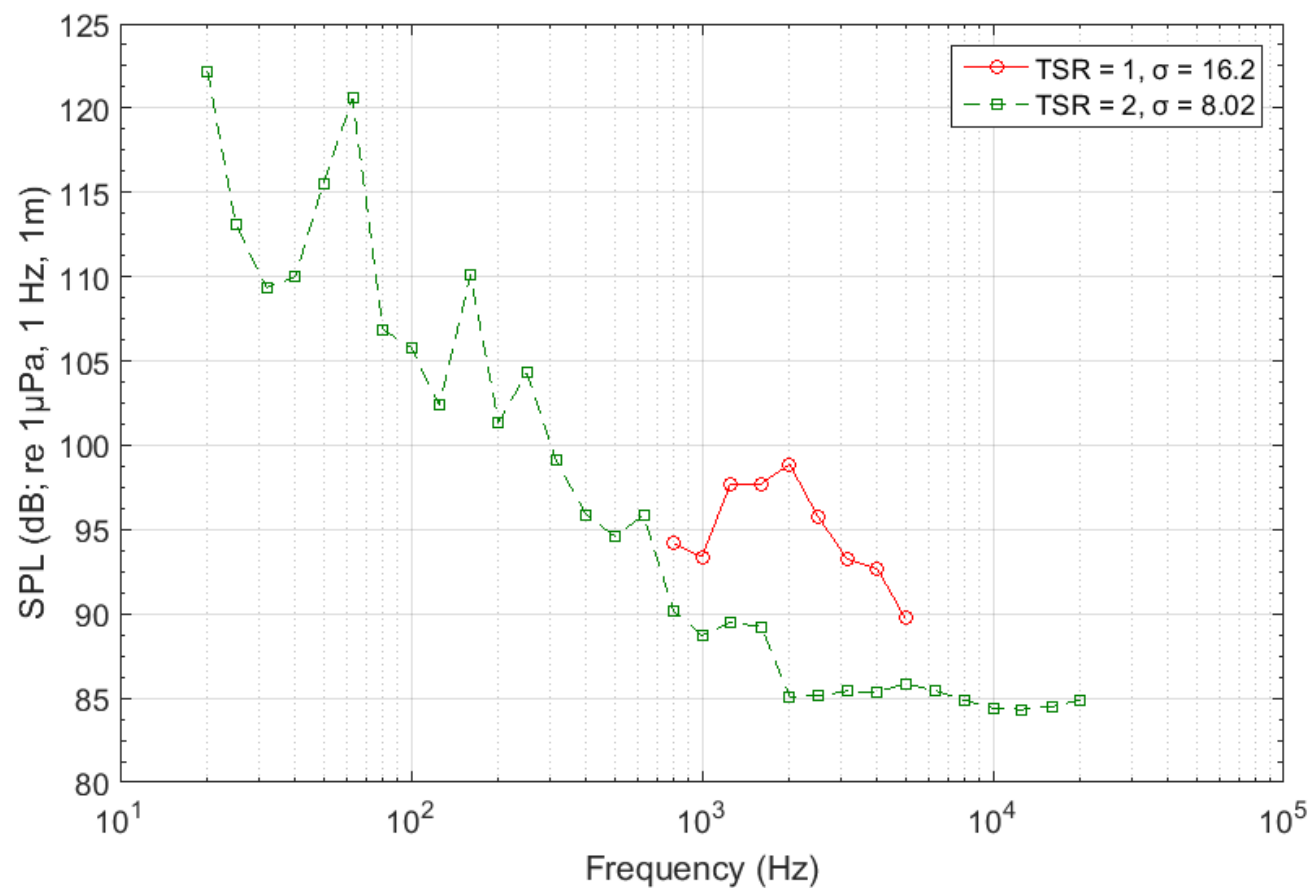

Fig. 12: Net 1/3 Octave Band Level revealed data only at $T S R=1$ and 2 for normal tunnel pressure

The noise surge that occurs at $T S R=1$ can be attributed to the presence of the intermittent cavitation. In addition, the thrust coefficient is the highest at this $T S R$, and the turbine is subjected to higher than normal axial loading. It is well known from the fundamentals of URN of a propeller that the effect of loading on a non-cavitating blade is one of the major contributors to the URN (Carlton, 2012). Typical horizontal axis turbines are subjected to axial loading with a maximum thrust coefficient between $0.8 \sim 1$ at optimal TSR (Bahaj et al, 2007; Johnstone et al, 2007). However, the Hydro-Spinna turbine produced a maximum thrust coefficient of 1.2 at $T S R=1$, which is not the optimal TSR in terms of the power performance. Interestingly, at the optimal TSR where maximum power coefficient is observed, there was no significant contribution from the turbine noise in the 1/3 Octave band level. 


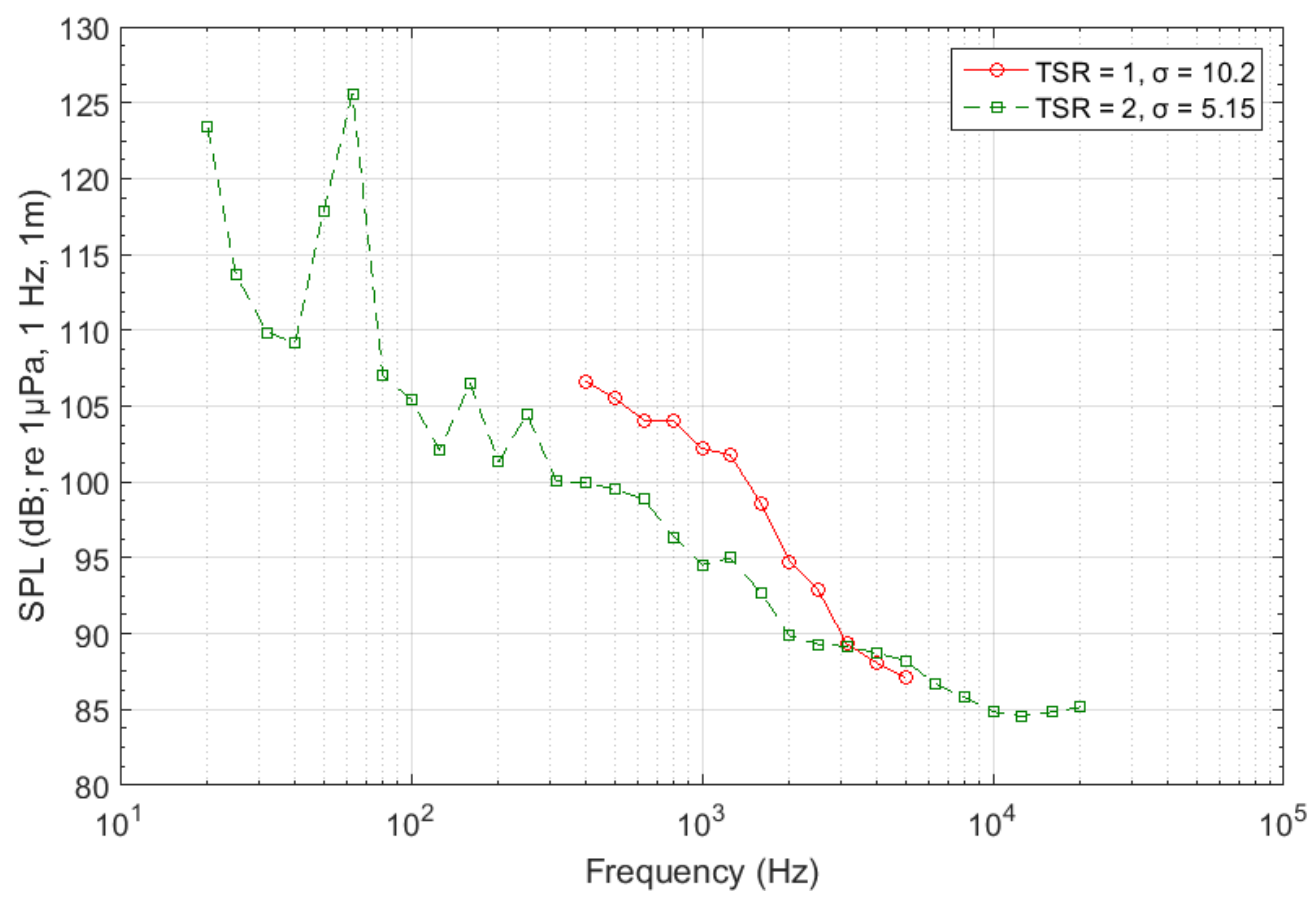

Fig. 13: Net 1/3 Octave Band Level for medium vacuum condition where weak tip vortex cavitation was observed

As the pressure was reduced to medium vacuum pressure, the turbine was observed to be cavitating at TSR 1 and 2. As a result, the SPL increased as the cavitation contributed to the URN of the turbine. At $T S R=1$, where cavitation was primarily observed, the cavitation noise dominated the mid frequency region between $400 \mathrm{~Hz}$ and $5000 \mathrm{~Hz}$, at SPL range of $106.5 \mathrm{~dB}$ to $87.5 \mathrm{~dB}$, as shown in Fig. 13. In comparison, at normal tunnel pressure the cavitation noise is in an SPL range of 99 to $90 \mathrm{~dB}$ with frequency range between $800 \mathrm{~Hz}$ and $5000 \mathrm{~Hz}$.

The tunnel pressure was further reduced to high vacuum pressure to induce cavitation and to investigate the cavitation behaviour of the Hydro-Spinna turbine. Fig. 14 presents the net 1/3 Octave data obtained for the test in which high SPL in the lower frequency region was recorded especially at TSR 1 and 2 where the cavitation was more obvious. As the TSR increased, the cavitation dissipated and the SPL level decreased. 


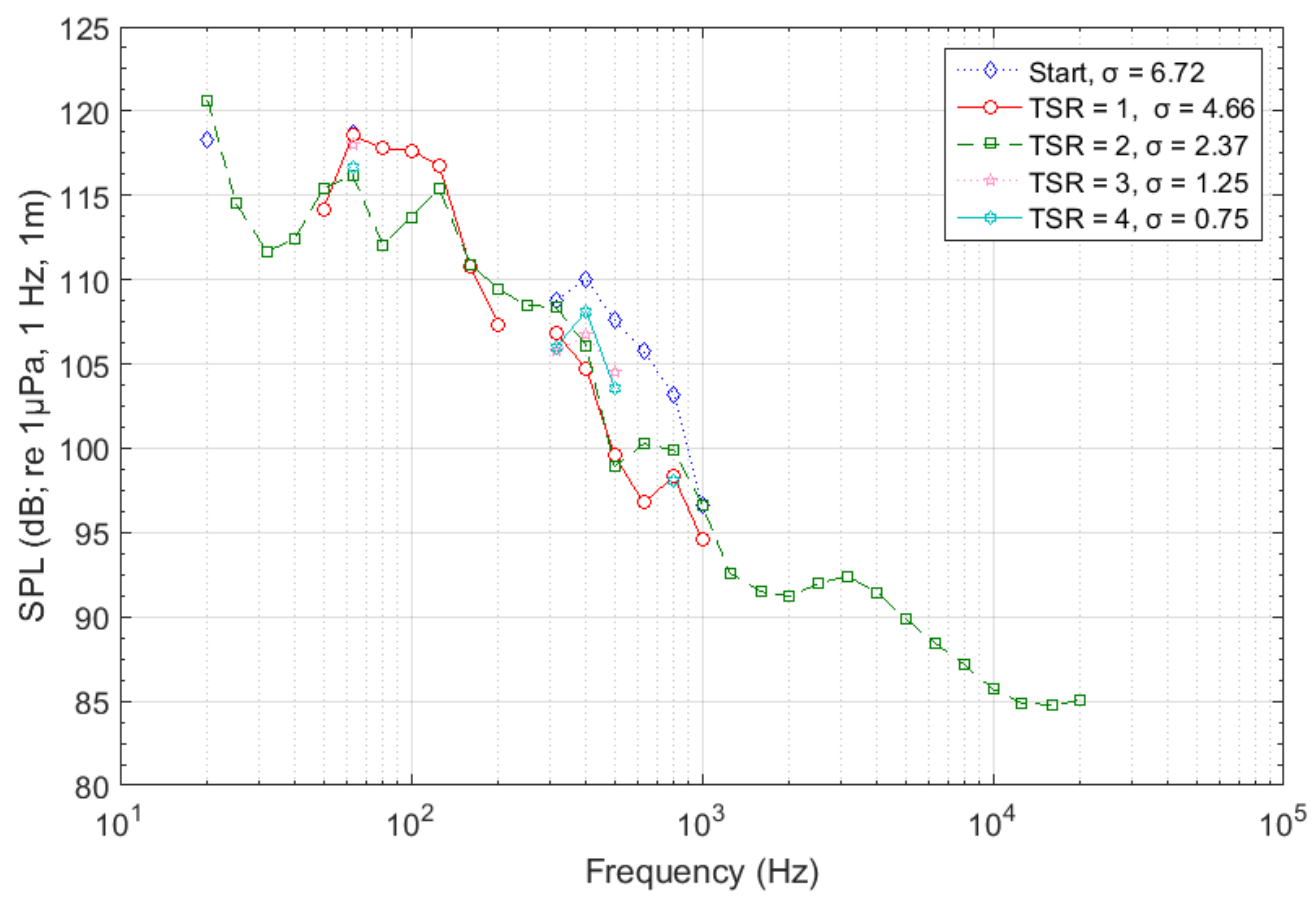

Fig. 14: Net 1/3 Octave Band Level for high vacuum condition where strong tip vortex cavitation was observed

It was also noted that at $T S R=1$, the cavitation noise dominated over the $T S R=2$ case in the low frequency region i.e. $50 \mathrm{~Hz}$ to $200 \mathrm{~Hz}$, where the turbine was cavitating more. However, in the mid frequency range i.e. $300 \mathrm{~Hz}$ to $1000 \mathrm{~Hz}$, the noise from $T S R=2$ is higher even though the turbine is cavitating less. This increase in SPL is believed to have been caused by the collapse of the cavitation bubbles as they travel in the slipstream and this was detected by the hydrophone. As observed, the turbine was cavitating more at $T S R=1$, in this condition, the tip vortex was strong due to the high axial load, hence the cavitation bubbles were able to travel along the slipstream and passed the hydrophone without collapsing. It was also observed that at $T S R=0$, high noise data was measured between $300 \mathrm{~Hz}$ and $1000 \mathrm{~Hz}$. It is believed that the turbine was cavitating in this condition, due to a combination of the low vacuum pressure and high axial loading, although no cavitation was visually detected in this condition. 


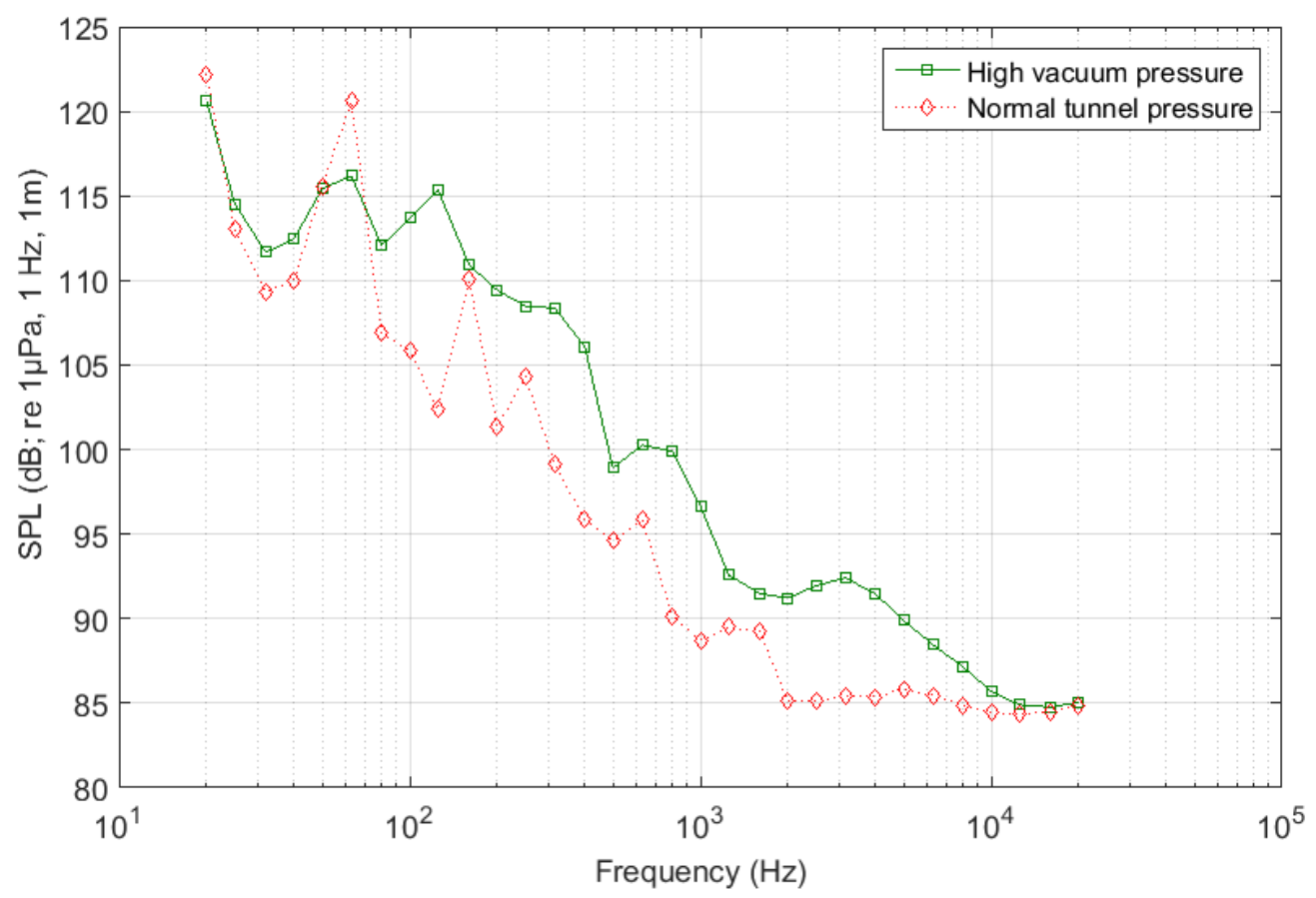

Fig. 15: Comparison of the Net 1/3 Octave Band Level for TSR $=2$ at high vacuum and normal tunnel pressures.

Fig. 15 presents a comparison at SPL at the same $T S R=2$, but at different tunnel pressures. At normal tunnel pressure, no cavitation was observed at this TSR while tip vortex cavitation were observed for the high vacuum pressure condition. This indicates that the presence of cavitation contributed to an increase in the URN generated by the turbine. At this $T S R=2$, the blade rate frequency was calculated to be $20.5 \mathrm{~Hz}$, and noise peaks are observed at its harmonic frequency. The noise peaks are more apparent in the normal tunnel pressure where no cavitation was observed whereas in the high vacuum pressure condition, the cavitation noise was more prominent.

\section{Full Scale Noise Level}

For propeller noise there are various methods reviewed in Atlar (Atlar et al, 2001) to predict the full scale noise level based on model scale measurements. It was also mentioned that accurate estimate of full scale noise based on model measurements is challenging without detailed knowledge of the model test facility. There was no correlation factor available for the Emerson Cavitation Tunnel, the facility where the model test was conducted. In the absence of such an in-depth scrutiny of the tunnel performance for noise, linear acoustical coefficients 
have been utilized for the extrapolation coefficients, as proposed by Starsberg (1977). The noise prediction was extrapolated by using the ITTC recommended scaling laws (Bertschneider et al, 2012; ITTC, 1987). It is important to note however, the ITTC scaling law is established to be used in propeller noise analysis for cavitating cases. The law takes into consideration different factors that affect the model scale measurement as well as the full scale operations.

Two extrapolation laws were considered in the noise analysis; they were the increase in noise level from model to full scale given in Equation 8 and the frequency shift in Equation 9:

$$
\begin{gathered}
\Delta L_{(F)}=20 \log \left[\left(\frac{D_{F}}{D_{M}}\right)^{z}\left(\frac{r_{M}}{r_{F}}\right)^{x}\left(\frac{\sigma_{F}}{\sigma_{M}}\right)^{y / 2}\left(\frac{n_{F} D_{F}}{n_{M} D_{M}}\right)^{y}\left(\frac{\rho_{F}}{\rho_{M}}\right)^{y / 2}\right] d B \\
\frac{f_{F}}{f_{M}}=\frac{n_{F}}{n_{M}}\left(\sqrt{\frac{\sigma_{F}}{\sigma_{M}}}\right)
\end{gathered}
$$

where subscripts $F$ and $M$ refers to full scale and model scale respectively, $D$ is the turbine diameter, $r$ is the reference distance at which the noise level is measured or predicted which is equal to $1 \mathrm{~m}$ in both cases, $\sigma$ is the cavitation number, $n$ is the turbine rotational speed and $\rho$ is the density of water which is $1002 \mathrm{~kg} / \mathrm{m}^{3}$ for the model test and the standard sea water value of $1025.9 \mathrm{~kg} / \mathrm{m}^{3}$ for the full scale turbine, while $y=2$ and $z=1$.

Full scale cavitation number was calculated using Equation 10:

$$
\sigma=\frac{P_{o}+\rho g\left(H_{S}-0.7 R\right)-P_{v}}{0.5 \rho W_{0.7}^{2}}
$$

where $H_{S}$ is the full scale immersion depth of the turbine from the water surface to turbine shaft.

Three full scale turbine diameters were considered in this approximation and their designed conditions are defined below: 
Turbine Diameter

Immersion Depth (surface to shaft)

Tidal Stream Velocity
$5 m, 10 m, 15 m$

$1 \times$ Turbine Diameter

$2 \mathrm{~m} / \mathrm{s}$

A propeller noise study was conducted in the same facility by Aktas et al (2016b) where model noise level was compared to that obtained from full scale trials using the same scaling laws. The general trend of the investigation was that the prediction underestimated the radiated noise level as compared to the full scale measurement especially in non-cavitating conditions. A similar methodology was also adopted by Wang et al (2007) in predicting the radiated noise level for a $12 \mathrm{~m}$ diameter turbine at designed tidal velocity of $3.5 \mathrm{~m} / \mathrm{s}$.

In the present study the reduced depth of immersion simulated shallow water and extreme wave conditions that would increase the risk of developing cavitating conditions. The radiated noise level prediction for the full-scale Hydro-Spinna turbines are intended to provide an indication of the real full scale conditions. Although there were no full scale levels to compare with as the turbines are still in the model development stage, the authors are confident of the prediction levels. The predicted 1/3 Octave band level at all TSR conditions for each full scale turbine is presented in Fig. 16-18.

The URN levels are plotted alongside the ICES noise level which is the fish reaction level threshold (Mitson, 1995). The ICES level is used in fisheries research vessels as to avoid disturbing the fish when conducting research. ICES effectively created a standard for the URN of research vessels. In the absence of even any remote standard regarding the fish response to sound, the ICES levels provide a good benchmark to compare against the extrapolated HydroSpinna URN levels. It offers an indication of the level of URN the Hydro-Spinna generated against a known fish threshold. ICES identified various fish species in their threshold and was discussed in detail by Mitson (2002). In addition, as it would be impossible to make the tidal turbines absolutely silent, evasion of the turbines would not be a problem. Considering a farm of tidal turbines with working at URN levels that are above the threshold of a number of fish 
species, this might mean that the aqua culture in an area may be disrupted. Therefore, in the case, ICES provides an approximate level to compare the turbine generated URN against.

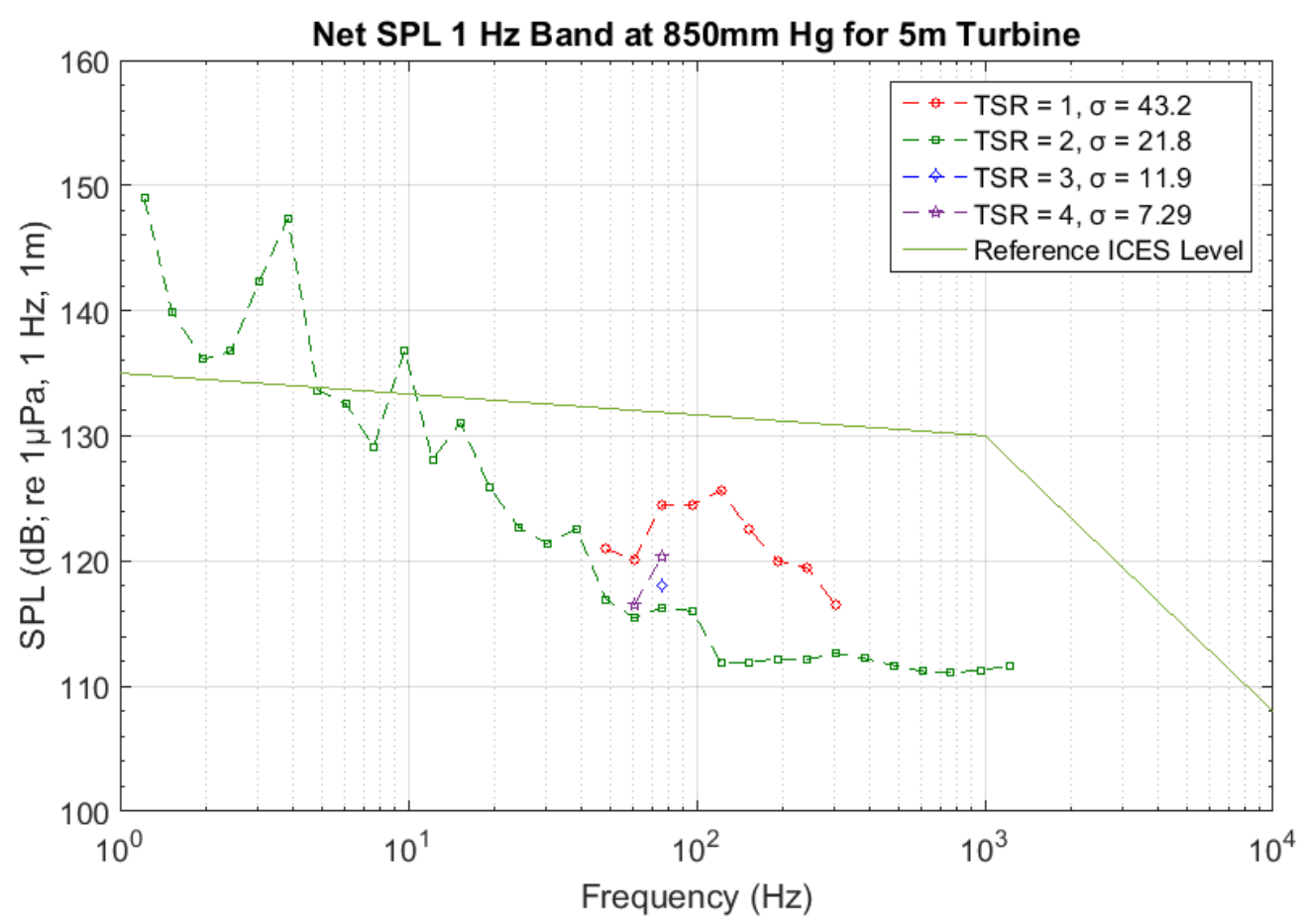

Fig. 16: Predicted noise level for 5m diameter full scale Hydro-Spinna turbine

It is observed for all full scale conditions, prominent net noise levels were only present at TSR 1 and 2 which is not the optimal TSR for the Hydro-Spinna operation. In this condition, a combination of the turbine blocking the flow and the consequent hydrodynamics forces resulting in the high thrust, caused increased flow separation that contributed to the significant increase in noise level. 


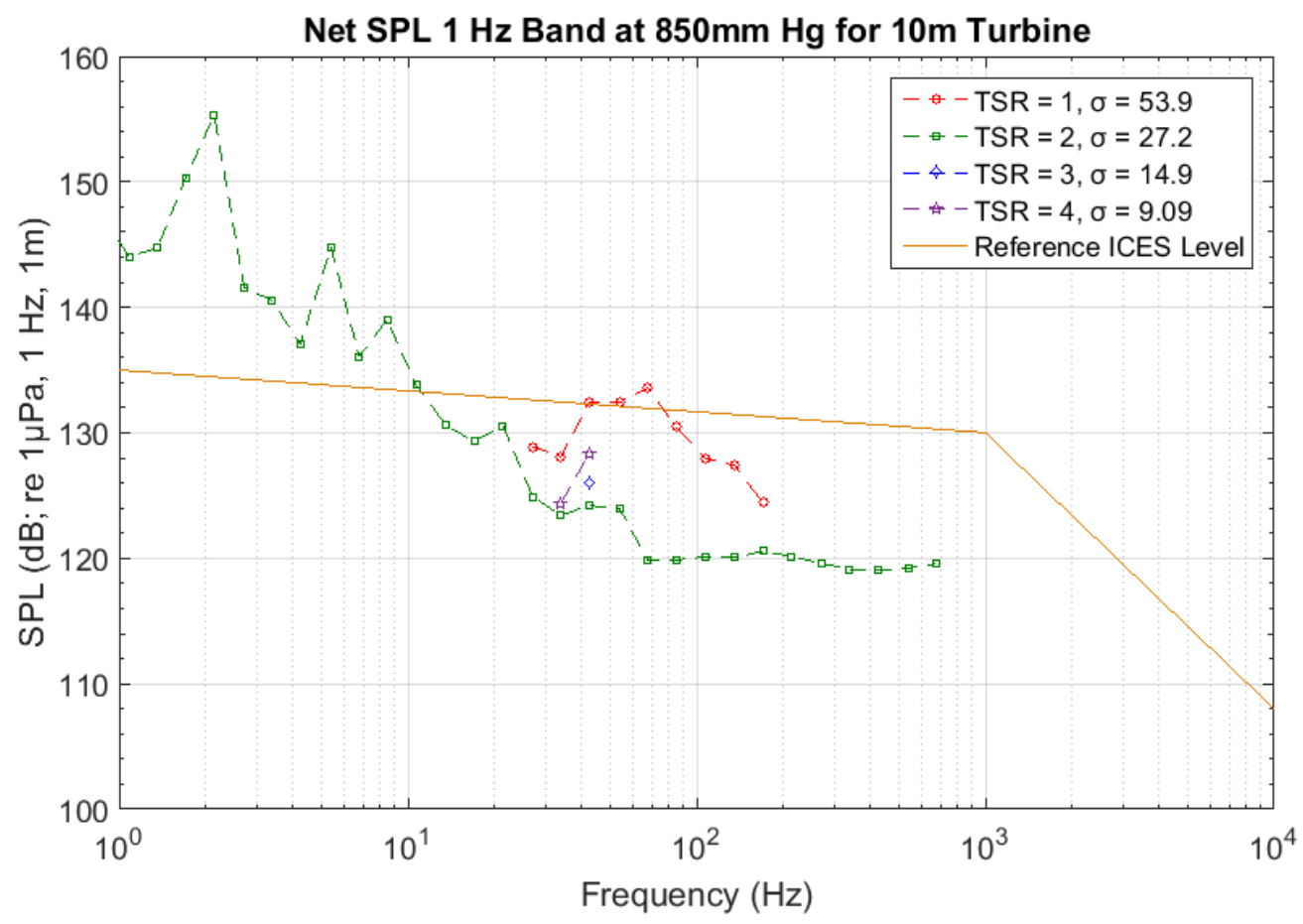

Fig. 17: Predicted noise level for 10m diameter full scale Hydro-Spinna turbine

For $T S R=1$, the noise level is observed at the mid frequency range between 20 and $120 \mathrm{~Hz}$. As the turbine diameter increases, the noise level increases but also shifted towards the lower frequencies. The same trend was observed with $T S R=2$ where a wider range of frequencies was obtained. More importantly, the turbines produced noise above the fish reaction level beginning at the lower frequency region where the highest noise level is observed. 


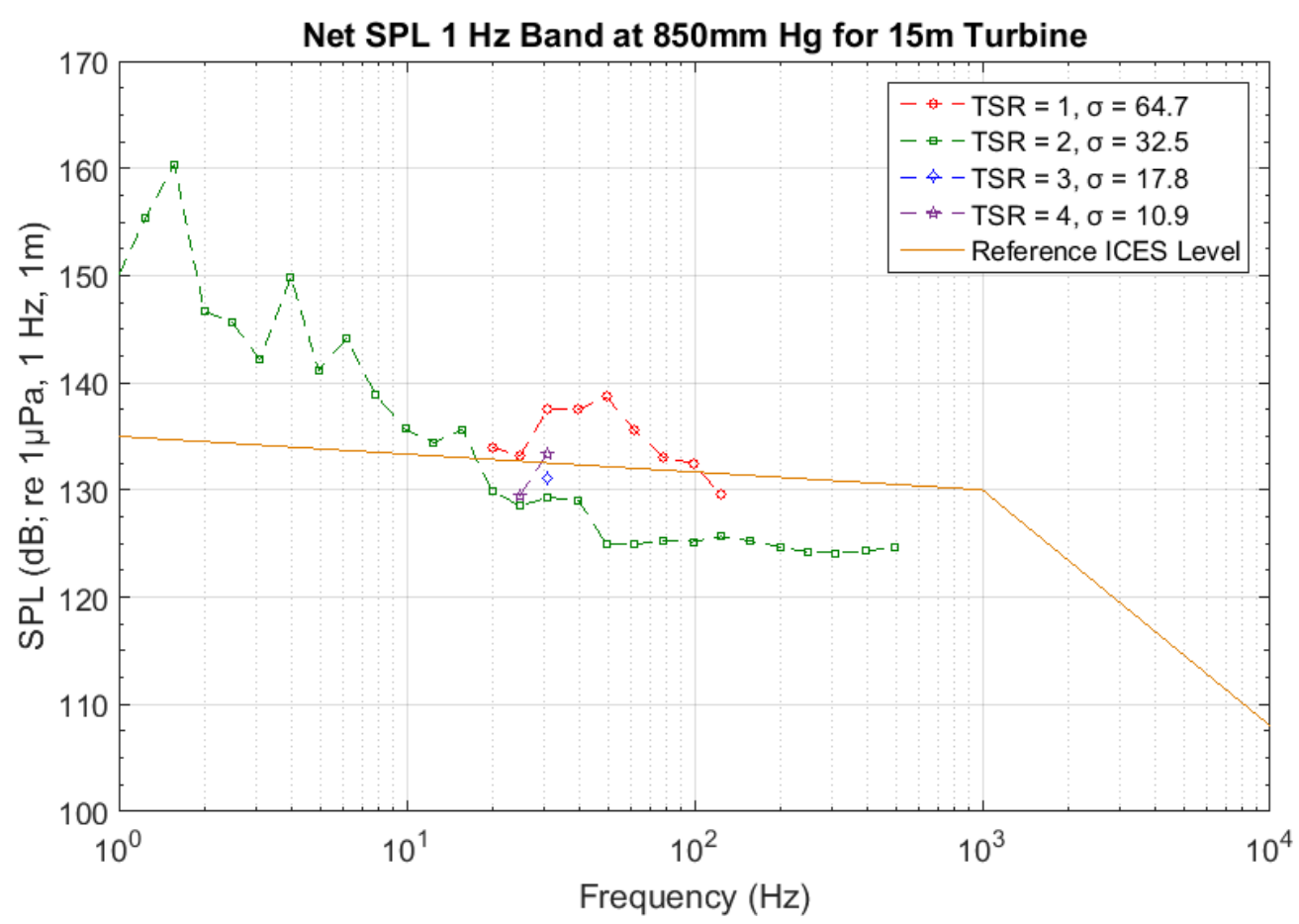

Fig. 18: Predicted noise level for 15m diameter full scale Hydro-Spinna turbine

With the largest diameter Hydro-Spinna, a maximum SPL of approximately $160 \mathrm{~dB}$ was estimated at a very low frequency of approximately $1.5 \mathrm{~Hz}$ at $T S R=2$ decreasing to $126 \mathrm{~dB}$ for higher frequencies between 250 to $500 \mathrm{~Hz}$. At the optimum TSR = 3, the maximum SPL produced is about $133 \mathrm{~dB}$ at a frequency of $31 \mathrm{~Hz}$.

\section{Conclusion}

The Hydro-Spinna was found to operate cavitation free in its optimal conditions and higher $T S R$. Weak intermittent tip vortex cavitation was observed at $T S R=1$ at normal tunnel pressure of $850 \mathrm{~mm} \mathrm{Hg}$. Vacuum pressure was introduced in the test to understand the cavitation behaviour of the turbine. For all tunnel pressure conditions, only tip vortex cavitation was observed during the tests and strongest tip vortex cavitation was detected at $T S R=1$ where the thrust coefficient of the turbine was the highest. The cavitation observation correlated very well against the noise measurement data where increased noise was measured when cavitation was visually detected. 
A full scale radiated noise level has been predicted by extrapolating experimental model scale data obtained from cavitation tunnel testing. The ITTC approved scaling law was used in this study for three different full scale turbine diameters for both cavitating and non cavitating cases. To date, no full scale Hydro-Spinna turbine has been developed, hence the extrapolated noise level could not be assessed against actual full scale measurements. Nonetheless, the results obtained provide a valuable overview on the magnitude of noise level likely to be radiated from full scale turbine operations and its potential impact on the marine environment. As background noise was eliminated in the analysis, the results presented are solely from the turbine hydrodynamic operation and flow characteristics of the marine current as well as cavitation for the cavitating case.

The Hydro-Spinna radiated noise results were plotted with ICES level as a reference where it was found that at certain off-design TSR, the turbines noise was higher than the threshold fish reaction level. This provides a good indicator of the potential noise level produced by the Hydro-Spinna turbine against the fish acoustic level. Nonetheless, at the optimal designed condition, the Hydro-Spinna was indicated to be operating below the fish reaction acoustic threshold.

\section{$\underline{\text { Acknowledgement }}$}

Authors would like to thank Michael Gilbert, the designer of the Hydro-Spinna, for his contribution. 


\section{$\underline{\text { References }}$}

Aktas, B., Atlar, M., Turkmen, S., Korkut, E. \& Fitzsimmons, P. (2016a) Systematic cavitation tunnel tests of a Propeller in uniform and inclined flow conditions as part of a round robin test campaign. Ocean Engineering, 120, 136-151.

Aktas, B., Atlar, M., Turkmen, S., Shi, W., Sampson, R., Korkut, E. \& Fitzsimmons, P. (2016b) Propeller cavitation noise investigations of a research vessel using medium size cavitation tunnel tests and full-scale trials. Ocean Engineering, 120, 122-135.

ANSI (2009) Quantities and Procedures for Description and Measurement of Underwater Sound from Ships - Part 1 General Requirements.

Atlar, M. (2011) Recent upgrading of marine testing facilities at Newcastle University, The 2nd International Conference on Advanced Model Measurement Technology for the EU Maritime Industry. Newcastle upon Tyne.

Atlar, M., Takinaci, A. C., Korkut, E., Sasaki, N. \& Takeshi, A. (2001) Cavitation tunnel tests for propeller noise of a FRV and comparisons with full-scale measurements, Fourth International Symposium on Cavitation. Pasadena, California.

Bahaj, A. S., Molland, A. F., Chaplin, J. R. \& Batten, W. M. J. (2007) Power and thrust measurements of marine current turbines under various hydrodynamic flow conditions in a cavitation tunnel and a towing tank. Renewable Energy, 32(3), 407-426.

Bailey, H., Senior, B., Simmons, D., Rusin, J., Picken, G. \& Thompson, P. M. (2010) Assessing underwater noise levels during pile-driving at an offshore windfarm and its potential effects on marine mammals. Marine Pollution Bulletin, 60(6), 888-897.

Bertschneider, H., Bosschers, J., Choi, G. H., Ciappi, E., Farabee, T., Kawakita, C. \& Tang, D. (2012) 27th ITTC Report of Specialist Committee on Hydrodynamic Noise, Copenhagen, Denmark.

Bevelhimer, M. S., Deng, Z. D. \& Scherelis, C. (2016) Characterizing large river sounds: Providing context for understanding the environmental effects of noise produced by hydrokinetic turbines. Journal of the Acoustical Society of America, 139(1), 85-92.

Brownell, B., Cato, D., Clark, C. W., Costa, D., Evans, P., Gedamke, J., Gentry, R., Giniser, B., Giordon, J., Jepson, P., Miller, P., Rendell, L., Tasker, M., Tyack, P., Vos, E., Whitehead, H., Wartzok, D. \& Zimmer, W. (2008) The effects of anthropogenic sound on marine mammals A draft research strategy.

Carlton, J. (2012) Marine Propellers and Propulsion, 3rd edition. Elsevier Ltd. 
EMEC (2013) European Marine Energy Centre, 2013. Available online: http://www.emec.org.uk/marine-energy/tidal-devices/ [Accessed.

Frisk, G. V. (2012) Noiseonomics: The relationship between ambient noise levels in the sea and global economic trends. Scientific Reports, 2.

Halvorsen, M. B., Carlson, T. J. \& Copping, A. E. (2011) Effects of Tidal Turbine Noise on Fish.

Hawkins, A., Hughes, D. \& Cheesman, S. (2008) Criteria and Metrics for Assessing the Effects of Underwater Sound on Fish and Invertebrates.

Hildebrand, J. A. (2009) Anthropogenic and natural sources of ambient noise in the ocean. Marine Ecology Progress Series, 395, 5-20.

ITTC (1987) 18th Report of Cavitation Committee, Kobe, Japan.

Johnstone, C. M., Grant, A. D., Connor, G. \& Clarke, J. A. (2007) Design and testing of a contra-rotating tidal current turbine. Proceedings of the Institution of Mechanical Engineers, Part A: Journal of Power and Energy, 221(2), 171-179.

Lloyd, T. P., Turnock, S. R. \& Humphrey, V. F. (2011) Modelling techniques for underwater noise generated by tidal turbines in shallow waters, Proceedings of the International Conference on Offshore Mechanics and Arctic Engineering - OMAE.

Lloyd, T. P., Turnock, S. R. \& Humphrey, V. F. (2013) Computation of inflow turbulence noise of a tidal turbine, 10th European Wave and Tidal Energy Conference. Aalborg, Denmark.

Lloyd, T. P., Turnock, S. R. \& Humphrey, V. F. (2014) Assessing the influence of inflow turbulence on noise and performance of a tidal turbine using large eddy simulations. Renewable Energy, 71, 742-754.

Madsen, P. T., Wahlberg, M., Tougaard, J., Lucke, K. \& Tyack, P. (2006) Wind turbine underwater noise and marine mammals: Implications of current knowledge and data needs. Marine Ecology Progress Series, 309, 279-295.

Mitson, R. B. (1995) Underwater Noise of Research Vessels: Review and Recommendations.

Mitson, R. B. (2002) Research Vessel Standards: Underwater Radiated Noise.

Molland, A. F., Bahaj, A. S., Chaplin, J. R. \& Batten, W. M. J. (2004) Measurements and predictions of forces, pressures and cavitation on 2-D sections suitable for marine current turbines. Proceedings of the Institution of Mechanical Engineers, Part M: Journal of Engineering for the Maritime Environment, 218(2), 127-138.

Park, C., Seol, H., Kim, K. \& Seong, W. (2009) A study on propeller noise source localization in a cavitation tunnel. Ocean Engineering, 36, 754 - 762. 
Pine, M. K., Jeffs, A. G. \& Radford, C. A. (2016) Effects of underwater turbine noise on crab larval metamorphosis. 2016. Available online: https://www.scopus.com/inward/record.uri?eid=2-s2.084948845642\&partnerID=40\&md5=84de37f74a6e953c433f392e9979015f.

Richardson, W. J., Greene, C. R., Jr., Malme, C. I., Thomson, D. H., Moore, S. E. \& Wiirsig, B. (2013) Marine Mammals and Noise.

Rosli, R., Norman, R. \& Atlar, M. (2016) Experimental investigations of the Hydro-Spinna turbine performance. Renewable Energy, 99, 1227-1234.

Rosli, R., Shi, W., Norman, R. \& Atlar, M. (2015) Cavitation tunnel investigation on the performance, cavitation and noise generation of marine current turbine: Hydro-Spinna, The 4th International Conference on Advance Model Measurement Technologies for the Maritme Industry. Istanbul, Turkey, 28-30 September 2015.

Ross, D. (1976) Mechanics of Underwater Noise. California,USA: Peninsula Publishing.

Shi, W., Atlar, M., Rosli, R., Aktas, B. \& Norman, R. (2016) Cavitation observations and noise measurements of horizontal axis tidal turbines with biomimetic blade leading-edge designs. Ocean Engineering, 121, 143-155.

Simmonds, M. P., Dolman, S. J., Jasny, M., Parsons, E. C. M., Weilgart, L., Wright, A. J. \& Leaper, R. (2014) Marine noise pollution - increasing recognition but need for more practical action. Journal of Ocean Technology, 9(1), 71-90.

SONIC (2012) Available [Downloaded

Spiga, I. (2014) Developing and testing models of fish behaviour around tidal turbines.

Spiga, I. (2016) Acoustic response to playback of pile-driving sounds by snapping shrimp. 2016. Available online: $\quad$ https://www.scopus.com/inward/record.uri?eid=2-s2.0$\underline{84948808992 \& \text { partnerID }=40 \& \mathrm{md} 5=\mathrm{e} 544 \mathrm{f} 529 \mathrm{e} 75895 \mathrm{~b} 87268 \mathrm{~d} 96 \mathrm{fe} 0 \mathrm{e} 10390 .}$.

Spiga, I., Cheesman, S., Hawkins, A., Perez-Dominguez, R., Roberts, L., Hughes, D., Elliott, M., Nedwell, J. \& Bentley, M. (2012) Understanding the Scale and Impacts of Anthropogenic Noise upon Fish and Invertebrates in the Marine Environment.

Starsberg, M. (1977) Propeller cavitation noise after 35 years of study, ASME Symposium on Noise in Fluids Engineering.

Thomsen, F., Mueller-Blenkle, C., Gill, A., Metcalfe, J., McGregor, P. K., Bendall, V., Andersson, M. H., Sigray, P. \& Wood, D. (2012) Effects of pile driving on the behavior of cod and sole. Advances in experimental medicine and biology, 730, 387-388.

Walsh, J., Bashir, I., Thies, P. R., Johanning, L. \& Blondel, P. (2015) Propeller cavitation noise after 35 years of study 
MTS/IEEE OCEANS 2015 - Genova: Discovering Sustainable Ocean Energy for a New World.

Wang, D., Atlar, M. \& Paterson, I. (2004) Performance Tests of the Second Tidal Stream Rotor.

Wang, D., Atlar, M. \& Sampson, R. (2007) An experimental investigation on cavitation, noise, and slipstream characteristics of ocean stream turbines. Proceedings of the Institution of Mechanical Engineers, Part A: Journal of Power and Energy, 221(2), 219-231.

Wenz, G. M. (1972) Review of Underwater Acoustics Research: Noise. Journal of the Acoustical Society of America, 51(3B), 1010-1024.

White, P. \& Pace, F. (2010) The impact of underwater ship noise on marine mammals, 1 st IMarEST Ship Noise and Vibration Conference.

Williams, R., Wright, A. J., Ashe, E., Blight, L. K., Bruintjes, R., Canessa, R., Clark, C. W., Cullis-Suzuki, S., Dakin, D. T., Erbe, C., Hammond, P. S., Merchant, N. D., O'Hara, P. D., Purser, J., Radford, A. N., Simpson, S. D., Thomas, L. \& Wale, M. A. (2015) Impacts of anthropogenic noise on marine life: Publication patterns, new discoveries, and future directions in research and management. Ocean \& Coastal Management, 115, $17-24$.

Wilson, B., Lepper, P. A., Carter, C. \& Robinson, S. P. (2014) Rethinking underwater soundrecording methods to work at tidal-stream and wave-energy sites., Marine Renewable Energy Technology and Environmental InteractionsSpringer. 